University of Louisville ThinkIR: The University of Louisville's Institutional Repository

Electronic Theses and Dissertations

$12-2015$

\title{
Evaluation of liver transplant prioritization methods for hepatocellular carcinoma patients using multistate models.
}

Sarah Katharine Alver

University of Louisville

Follow this and additional works at: https://ir.library.louisville.edu/etd

Part of the Public Health Commons

\section{Recommended Citation}

Alver, Sarah Katharine, "Evaluation of liver transplant prioritization methods for hepatocellular carcinoma patients using multistate models." (2015). Electronic Theses and Dissertations. Paper 2331.

https://doi.org/10.18297/etd/2331

This Master's Thesis is brought to you for free and open access by ThinkIR: The University of Louisville's Institutional Repository. It has been accepted for inclusion in Electronic Theses and Dissertations by an authorized administrator of ThinkIR: The University of Louisville's Institutional Repository. This title appears here courtesy of the author, who has retained all other copyrights. For more information, please contact thinkir@louisville.edu. 


\title{
EVALUATION OF LIVER TRANSPLANT PRIORITIZATION METHODS FOR HEPATOCELLULAR CARCINOMA PATIENTS USING MULTISTATE MODELS
}

\author{
By \\ Sarah Katharine Alver \\ B.S., Utah State University, 2001
}

\begin{abstract}
A Thesis Submitted to the Faculty of the School of Public Health and Information Sciences of the University of Louisville in Partial Fulfillment of the Requirements for the Degree of

Master of Science

in Biostatistics: Decision Science

Department of Biostatistics and Bioinformatics

University of Louisville

Louisville, KY
\end{abstract}

December 2015 



\title{
EVALUATION OF LIVER TRANSPLANT PRIORITIZATION METHODS FOR HEPATOCELLULAR CARCINOMA PATIENTS USING MULTISTATE MODELS
}

\author{
By \\ Sarah Katharine Alver \\ B.S., Utah State University, 2001
}

A Thesis Approved on

November 18, 2015

By the Following Thesis Committee:

Guy Brock, PhD

Michael Marvin, MD

Douglas Lorenz, PhD

Maiying Kong, $\mathrm{PhD}$ 


\section{ACKNOWLEDGEMENTS}

I would like to thank my major professor, Dr. Guy Brock, and my committee members Dr. Michael Marvin, Dr. Douglas Lorenz and Dr. Maiying Kong for their valuable input and time. I also thank Dr. Brock and Dr. Marvin for the opportunity to participate in this research. As well, I thank Dr. Brock for his teaching and willingness to share his ideas and enthusiasm. I have learned a great deal from this project and could not have done so without his guidance and patience. Additionally, I thank my husband John and my son Gabriel for their help and understanding during the past two years. I could not have completed this project or this degree if John had not taken on so much more than his share of our responsibilities during this time or if Gabriel were not such a reasonable and good-natured teenager. Finally, I thank my coworkers and friends, Phyllis Spalt and Anna Hartman, for their encouragement and support. 


\title{
ABSTRACT \\ EVALUATION OF LIVER TRANSPLANT PRIORITIZATION METHODS FOR HEPATOCELLULAR CARCINOMA PATIENTS USING MULTISTATE MODELS
}

\author{
Sarah K. Alver
}

November 18, 2015

The Model for End Stage Liver Disease (MELD), used for prioritizing liver transplantation, predicts mortality from liver disease. Patients with hepatocellular carcinoma $(\mathrm{HCC})$ risk disease progression not reflected in their MELD score. Exception scores prioritize HCC patients higher than their MELD scores, but advantage them over non-HCC patients. To address this, a delay of six months for using exception scores has been implemented, and alternative HCC-specific scores have been developed. Using multistate models, this study projects waitlist dropout and transplant probabilities under the delay and under two alternative scores. The delay improves equity between HCC and non-HCC patients for the first six months waitlist time, but still advantages HCC patients after six months. Both alternative scores would improve this inequity but increase dropout for some $\mathrm{HCC}$ risk groups and decrease $\mathrm{HCC}$ transplant probabilities below non-HCC probabilities. Further calibration of these scores is recommended prior to considering them for implementation. 


\section{TABLE OF CONTENTS}

PAGE

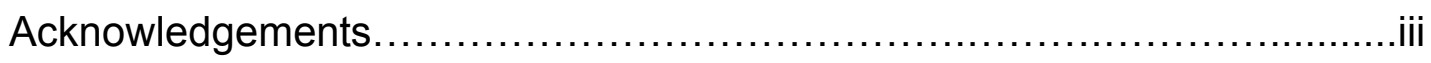

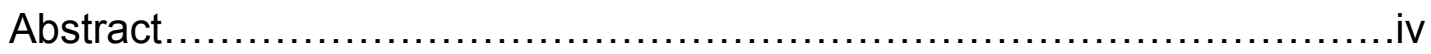

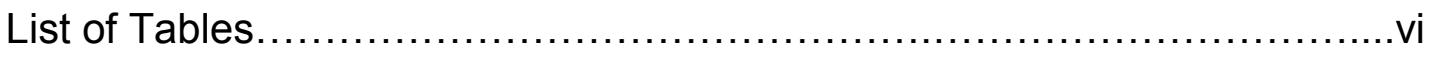

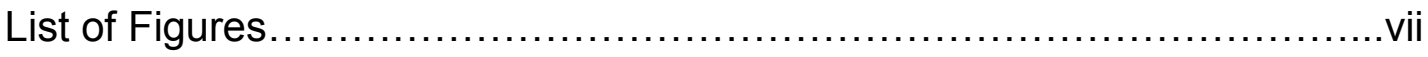

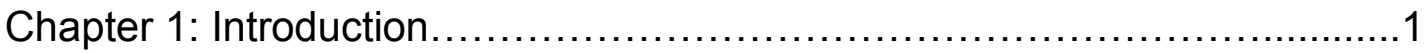

Chapter 2: Six-Month Delay and MELD ${ }_{\mathrm{EQ}}$ Comparison.................. 15

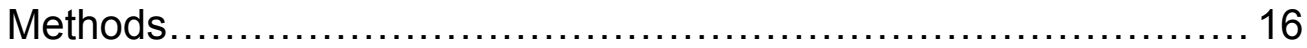

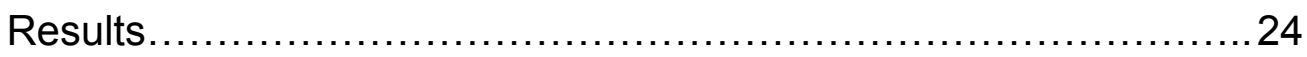

Discussion................................................ 33

Chapter 3: MELD ${ }_{\mathrm{EQ}}$ and deMELD Comparison $\ldots \ldots \ldots \ldots \ldots \ldots \ldots \ldots \ldots \ldots \ldots \ldots$

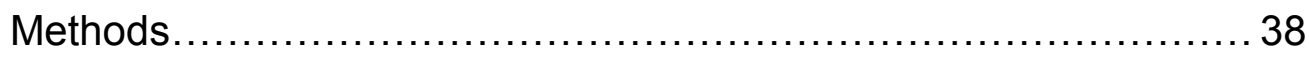

Results..................................................... 42

Discussion............................................... 58

Chapter 4 : Summary ............................................. 64

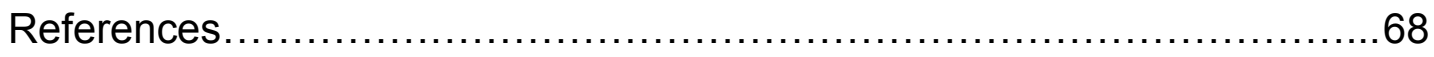

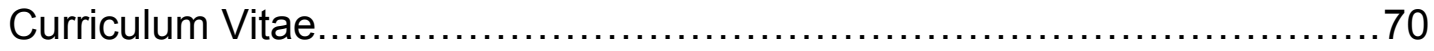




\section{LIST OF TABLES}

TABLE

PAGE

1. Outcomes at last follow up for HCC and non-HCC patients............... 24

2. Frequency distributions of the MELD $D_{E Q}$ and laboratory MELD scores for HCC patients at initial listing.

3. Overall current and projected $\mathrm{HCC} /$ non-HCC dropout/transplant probabilities

4. Frequency distributions of MELD $D_{E Q}$ and deMELD scores at listing

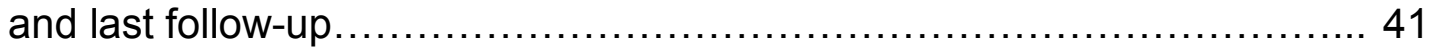

5. Differences in projected HCC dropout and actual non-HCC dropout....... 45

6. Cross-tabulation of equivalent MELD score assignments.................53

7. Effect of covariates with score agreement.............................. 54

8. Effect of covariates without score agreement............................ 55

9. Hazard Ratios for post-transplant survival............................. 57 


\section{LIST OF FIGURES}

FIGURE

PAGE

1. Diagram of multistate model, five groups............................... 23

2. Actual/projected dropout/transplant stratified by laboratory MELD..........30

3. Actual/projected dropout/transplant stratified by MELD $\mathrm{EQ}_{\mathrm{EQ}}(\mathrm{HCC})$ and laboratory MELD (non-HCC), time since listing ....................... 31

4. Actual/projected dropout/transplant stratified by MELD $D_{\mathrm{EQ}}(\mathrm{HCC})$ and laboratory MELD (non-HCC), 6-18 months from listing ................. 32

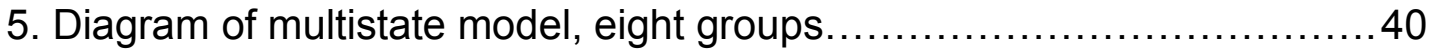

6. Actual/projected dropout/transplant stratified by MELD $\mathrm{EQ}_{\mathrm{EQ}}(\mathrm{HCC})$ and laboratory MELD (non-HCC) $<22$, time since entering state.

7. Actual/projected dropout/transplant stratified by MELD $\mathrm{EQ}_{\mathrm{E}}(\mathrm{HCC})$ and laboratory MELD (non-HCC) $\geq 22$, time since entering state

8. Actual/projected dropout/transplant stratified by deMELD (HCC) and laboratory MELD (non-HCC) <22, time since entering state.

9. Actual/projected dropout/transplant stratified by deMELD (HCC) and laboratory MELD (non-HCC) $\geq 22$, time since entering state.

10. Post-transplant survival for patients stratified by MELD $D_{E Q}$ and deMELD 


\section{CHAPTER 1 \\ INTRODUCTION}

The Model for End-Stage Liver Disease (MELD) score, which predicts three-month mortality from liver disease, has been used to allocate liver transplants to patients with the most need or urgency for transplant under policies of the Organ Procurement and Transplantation Network (OPTN). The organ transplant system is managed in the Unites States through these policies by the United Network for Organ Sharing (UNOS) under contract with the federal government. The MELD score was originally used to predict three-month mortality for patients receiving TIPS (Transjugular Intrahepatic Portosystemic Shunt) procedures ${ }^{1}$, and subsequently generalized to predict three-month mortality risk in patients with end-stage liver disease ${ }^{2}$.

The MELD score is based on the biochemical values of bilirubin, international normalized ratio (INR) and creatinine. It is defined by:

$$
0.957^{*} \ln (\text { creatinine })+0.378^{*} \operatorname{In}(\text { bilirubin })+1.12^{*} \ln (I N R)+0.643
$$

where creatinine and bilirubin are in milligrams per deciliter $(\mathrm{mg} / \mathrm{dL})$. The result of this calculation is rounded to the tenth decimal place and then multiplied by 10 . The minimum of this score is 6 , and the maximum is 40 . Laboratory values less than 1 are set to 1 for the calculation, and creatinine is set to 4 for candidates with creatinine greater than 4 or those who received two or more dialysis treatments or 24 hours of continuous veno-venous hemodialysis within the prior week $^{3}$. 
Liver transplant has been shown to be an appropriate treatment for patients with hepatocellular carcinoma whose tumor characteristics meet certain criteria for size and number. These criteria, a single tumor $5 \mathrm{~cm}$ or less in diameter or up to 3 tumors $3 \mathrm{~cm}$ or less in diameter, have been referred to as the Milan criteria ${ }^{4}$. Patients with hepatocellular carcinoma (HCC) often have higher mortality risk than their calculated MELD scores would indicate due to tumor progression rather than liver disease ${ }^{5}$, and also risk of dropout from the waiting list due to HCC progression beyond criteria. In attempt to account for this, HCC patients have been allocated exception MELD scores per OPTN policies which are generally greater than their calculated MELD score. However, risk of tumor progression beyond the Milan criteria within three months was later found to be overestimated by these exception points ${ }^{6}$, such that HCC patients have had higher transplant rates and lower waitlist dropout rates than non-HCC candidates. Thus, policies have been revised several times - for example, candidates with single tumors less than $2 \mathrm{~cm}$ in size were originally granted exception points but currently are not. Under recent policy, candidates with HCC who met criteria according to OPTN policies received an initial exception MELD score equivalent to a 15 percent risk of 3-month mortality ${ }^{7}$. Criteria in the OPTN policies include stage T2 lesions, which may be one lesion greater than or equal to $2 \mathrm{~cm}$ and less than or equal to $5 \mathrm{~cm}$ in size, or two or three lesions greater than or equal to $1 \mathrm{~cm}$ and less than or equal to $3 \mathrm{~cm}$ in size. Additional exception points equivalent to a $10 \%$ increase in mortality risk were given every three months thereafter, until the patient received a transplant or became unsuitable for transplant based on their HCC progression ${ }^{7}$. 
Under this system, despite previous modifications, patients with HCC continued to have an advantage for access to transplantation when compared to patients without $\mathrm{HCC}^{5,8}$. Because previous policies provided advantages to HCC patients compared to non-HCC patients, with increased transplant rates and lower dropout rates ${ }^{9}$, OPTN/UNOS recently implemented a six-month delay before granting exception points ${ }^{3}$. Simulation modeling previously suggested that this delay would help decrease disparity between HCC and non-HCC candidates ${ }^{10}$. With the six-month delay, HCC patients will be listed at their laboratory MELD score until the second three month extension. At that time they will receive an exception MELD score equivalent to a $35 \%$ risk of 3-month mortality (score of 28) and then continue with the scheduled exception point progression every three months until they receive transplantation or become unsuitable based on HCC progression. The exception point progression will be capped at 34 points ${ }^{3}$.

In addition to the six-month delay, investigators have proposed alternative scoring models for HCC patients in attempt to address the disparity in access to transplantation between HCC and non-HCC patients ${ }^{6,11-15}$. These scores were intended to more accurately reflect dropout risk for $\mathrm{HCC}$ patients based on $\mathrm{HCC}$ and patient characteristics in addition to the laboratory MELD. Several of these scores were summarized in a previous publication ${ }^{16}$ and those models as well as another mode ${ }^{15}$ that was developed more recently will be briefly described here.

\section{Review of Equivalent MELD Scores}

One of the alternative HCC transplant prioritization scores, developed by Freeman et al, estimates 90-day dropout probability for HCC patients using an 
equation based on calculated MELD score at listing, maximum tumor size, and alpha-fetoprotein (AFP) ${ }^{6}$, a laboratory test used by clinicians in screening for and monitoring $\mathrm{HCC}^{17}$. The dropout probability from the equation can then be matched to the non-HCC MELD score with that same dropout probability. They developed this equation by examining multiple covariates using Cox regression models with the endpoint of time to dropout. Their data included 11431 patients, 2052 of whom received exceptions due to $\mathrm{HCC}$, on the waiting list between $4 / 30 / 2003$ and $12 / 31 / 04$. They found that MELD at listing, maximum tumor size, AFP and age at listing were associated with an increased relative risk of waitlist removal (dropout), while ablation, diagnostic imaging modality (magnetic resonance imaging or ultrasound vs computerized tomography), number of tumors, race, gender and blood type did not have significant relative risks for removal. They purposefully used only nondiscriminatory variables in the equation, so age was excluded. They assessed the accuracy of their equation with newer waiting list data from 1/1/2005 to 4/20/2005 that included 624 HCC candidates and found that the concordance of their model was $0.781(95 \% \mathrm{Cl}$ $0.688,0.853)$. However, MELD alone was equally predictive of dropout with concordance of $0.796(95 \% \mathrm{Cl} 0.657,0.897)$. Additionally, this model was validated in a separate study in 2008 on $390 \mathrm{HCC}$ transplant candidates who received locoregional therapy. This study found concordance of $0.81(0.685$, 0935) at three months and $0.8(0.732,0.868)$ at six months for this model's accuracy in predicting tumor progression. For this group of patients, the model was more predictive than MELD alone, which had concordance of $0.574(0.420$, $0.728)$ at three months and $0.538(0.413,0.663)$ at six months ${ }^{18}$. The authors of 
the validation study noted that the patients undergoing locoregional therapy had low baseline MELD scores that could be a reason for the contrast with the original study, which found that MELD was equally predictive of dropout. Advantages of this score include that it was externally validated and it also identified risk factors for HCC waitlist dropout. Disadvantages are that it estimates dropout probability rather than an actual score, so may be difficult to implement in practice. Also, although it was validated, it was not found to be better than MELD alone in predicting dropout when validated on a larger, broader group of patients.

Another score that has been developed is that by Piscaglia et al. This score adds points to the lab MELD for waitlist time in months and a "stage score" that incorporates tumor size and consideration for downstaging ${ }^{11}$. The stage score included 5 points for a single nodule up to $3 \mathrm{~cm}$ in size, 8 points for larger tumors or multifocal tumors within the Milan criteria, or 12 points for downstaging protocol. The authors stated that the number of points chosen were arbitrary, but did increase with increasing disease stage to reflect higher risk of dropout. The downstaging protocol in this study allowed patients with $\mathrm{HCC}$ previously beyond Milan criteria who underwent surgery or locoregional treatment to be listed for transplant if the tumor size was reduced to within Milan criteria and their AFP level did not exceed 400 nanograms per milliliter $(\mathrm{ng} / \mathrm{mL})$ during listing. During the time of the study, the Bologna Transplantation Center in Italy adopted a MELD-based prioritization policy (UNOS also began using the MELD in 2002 along with exception points for HCC patients), but used its own allocation policy, which was the score described above, for patients with HCC. The patients in the 
study included $301 \mathrm{HCC}$ and non-HCC patients listed for transplant at the Bologna Transplantation Center from 3/1/2001 to 2/28/2004, including 163 patients listed before the policy implementation and 138 listed after. They compared dropout and transplant rates between $\mathrm{HCC}$ and non-HCC patients before implementing this policy and after it had been in place for one year. They did not find a difference in dropout between the two time periods, but HCC patients had a significantly higher cumulative probability of transplant, assessed using Kaplan-Meier curves, compared to non-HCC patients after implementing the new score. This transplant probability was also significantly higher compared to HCC probabilities before implementing their new score, and they did not find this difference for non-HCC patients. There was concern that the transplant rates for $\mathrm{HCC}$ were excessively greater than those for non-HCC patients when the policy was first implemented. However, the authors stated that when their policy was first implemented many HCC patients initially received priority due to their long waiting time, but after these initial patients underwent transplantation the rates became more equal between $\mathrm{HCC}$ and non-HCC patients. They also pointed out that non-HCC dropout rates did not significantly increase; however, the proportion of non-HCC patients still waiting for transplant at the end of the study period did increase. Based on this, the policy at that center was updated to provide fewer points: 3 points for a single tumor up to $3 \mathrm{~cm}$ with 0.5 points per month waiting time and 6 points for larger tumors, multiple tumors or downstaging protocol with 1 point per month waiting time. Results of this updated score were not included in the study. Advantages of this score include that it does consider HCC characteristics in addition to MELD. Another 
advantage, in theory, is that it was updated based on results, reflecting the idea that patient and center characteristics can change over time, though results of the update are not known. Other authors have stated that allocating increased points for downstaging is controversial as these patients may have decreased dropout risk $^{16}$; also a waiting period before transplant may be recommended ${ }^{19}$. While downstaging protocols are used in some regions in the United States ${ }^{19,20}$, automatic exception points for downstaged tumors are generally not given ${ }^{3,20}$, so implementing a score that gives increased points for downstaging in the US may be difficult.

Another equivalent MELD score, developed by Vitale et al ${ }^{14}$, was derived using transplant benefit rather than dropout risk as an endpoint to equate with that of non-HCC patients. This is in contrast to several of the other scores reviewed here which equate to non-HCC using dropout risk. It also includes a separate utility score intended to avoid poor post-transplant outcomes. The authors state that using 3-month dropout risk as an endpoint for both $\mathrm{HCC}$ and non-HCC patients may be problematic because dropout for non-HCC patients is usually due to death, whereas dropout for HCC patients is usually due to tumor progression. To derive their score, the investigators studied covariates using competing risk models for analyzing waitlist outcomes, to allow for the outcomes of transplant, death or still waiting, and Cox proportional hazard regression models for analyzing transplant outcomes. They studied 2697 patients who were placed on the transplant waiting list and 1702 who were undergoing liver transplant during 2004-2009 in the North Italy Transplant Program area. The final covariates used in the score were laboratory MELD and AFP. The paper notes 
that AFP was associated with death on the waitlist for HCC patients, but had a more significant impact on post-transplant survival. Monthly death probabilities were obtained from the competing risk and Cox models, and then used to simulate 1000 outcomes (life expectancy in months based on the covariates) for both $\mathrm{HCC}$ and non-HCC groups. Then a regression model was used to estimate the five-year transplant benefit based on MELD for non-HCC patients and MELD and AFP for HCC patients, and these were equated between the two groups to obtain an equivalent MELD score. Thus, the HCC transplant survival benefit for a given equivalent MELD score would be equal to that of non-HCC patients with the same numerical value for MELD. They also developed a utility score that, expressed as a linear relationship between $\log _{10}$ AFP and MELD, calculates the maximum MELD a patient may have with any given AFP value (or vice versa) to prevent unacceptable post-transplant outcomes, which were defined as less than $50 \%$ survival at five years. Another difference between this score and others reviewed here is that the term for AFP is negative (so a patient will receive fewer points for a higher AFP), which follows from their findings that AFP had a more significant impact on post-transplant survival and their use of survival benefit as the point to equate with non-HCC MELD scores. An advantage of this score is that it does consider both dropout and post-transplant survival. A disadvantage may be that AFP is the only HCC-specific characteristic included in the model, where other investigators have found that additional HCC-related characteristics such as tumor size can influence dropout risk ${ }^{5,6}$. The authors state that the score needs external validation and further refinement. 
Toso et al developed an HCC scoring model called the dropout equivalent MELD in 2012 that included age, laboratory MELD, tumor size, In(AFP), number of tumors, diagnosis (additional causes of liver disease including alcohol, hepatitis $C$ virus, hepatitis $B$ virus, hemochromatosis, non-alcoholic steatohepatitis and "other") in the prediction of three-month dropout probability ${ }^{12}$. The coefficients were positive for all the HCC-related covariates in the score as well as for age. In deriving this model, the investigators used OPTN data from January 2004 to December 2009 including 5498 HCC patients and 43528 nonHCC patients. They restricted age to $\geq 45$ years. Other variables explored included total tumor volume. They used multistate modeling, competing risks analysis and linear regression to identify covariates associated with three-month dropout probability and to model that probability as a function of them. They derived an equation estimating a logit transformation of dropout probability as a function of the covariates for each group, $\mathrm{HCC}$ and non-HCC, and then set the $\mathrm{HCC}$ and non-HCC equations equal to each other to obtain the dropout equivalent MELD. The paper notes that all patients with $\mathrm{HCC}$ are given the same number of exception points at each time point under the current policy. Though most are given a higher score than their dropout probability warrants, some HCC patients would be underserved by receiving a lower score than their dropout probability would indicate based on their HCC characteristics. Advantages of this score include that it incorporates several HCC-related covariates, which were previously established as risk factors for dropout, and is on the same scale as the laboratory MELD. Disadvantages include the potential ethical concern of including age in the model as well as possible worse post- 
transplant outcomes for older patients, and also the potential of higher scoring of patients with aggressive tumors who may have higher risk of $\mathrm{HCC}$ recurrence post-transplant. The paper notes that prospective assessment and external validation of the model could address this along with a minimum 3-month wait before transplantation if needed, to exclude those with high risk of post-transplant recurrence. The authors also noted the need for ongoing assessment and updating of such a model; for example, adjustment for newly identified risk factors for dropout or changes in other HCC treatments.

In 2014, Toso et al developed a new equivalent MELD score that uses laboratory MELD, number of tumors, AFP, and tumor size ${ }^{13}$. This model was developed using a training set of 49026 patients from the United States, listed between 1/1/2004 and 12/31/2009, and validated on two datasets, one with 20475 United States patients (listed 1/1/2009 to 12/31/2011) and the other with 1781 United Kingdom patients (listed 7/1/2008 to 9/30/2011). The coefficients for all the covariates in this equivalent score are positive. The score uses discrete cutoff points, noted by the authors to be clinically relevant, for all the covariates except laboratory MELD. This is discussed further in Chapter 3. In contrast, their previous score used continuous values for most covariates. For this model, the investigators decided not to use age and type of liver disease since these factors are not used when prioritizing non-HCC patients. Similarly to the model they developed in 2012 described earlier, this model was based on three-month dropout probability, which was modeled using the covariates listed above and then equated to the three-month dropout probability of non-HCC patients to obtain an equivalent HCC MELD score. They termed this score the "new dropout 
equivalent MELD (deMELD)". Using the validation data sets, they validated the resulting score as well as the exception MELD score, laboratory MELD, and a variation of their score which takes the maximum of the calculated new deMELD and the laboratory MELD (termed "mixed new deMELD"). The concordance indices for the mixed new deMELD were $0.67(95 \% \mathrm{Cl} 0.622,0.714)$ in the US validation set and $0.652(95 \% \mathrm{Cl} 0.553,0.745)$ in the UK validation set, compared to $0.566(0.532,0.597)$ and $0.527(0.483,0.594)$ respectively for the exception MELD and $0.663(0.616,0.707), 0.657(0.56,0.75)$ respectively for the laboratory MELD. The authors recommended that the score be updated at least every 3 months during listing, and this would capture improvements due to other therapies such as ablation (e.g. a smaller tumor size after treatment would result in a lower deMELD score). Again, they noted that the score could lead to transplantation of patients with more aggressive $\mathrm{HCC}$ that risk post-transplant recurrence, and suggested considering a 3-6 month waiting time to help control this risk. They also discussed the potential of building a model based on transplant benefit rather than or in addition to dropout risk.

The MELD $D_{E Q}$, derived by Marvin et al in $2015^{15}$, includes laboratory MELD, In(AFP), number of tumors, maximum tumor size, and a constant that is increased after six months on the waiting list. The authors derived this score using OPTN data from $1 / 22 / 2005$ to $9 / 30 / 2009$, including 7289 HCC patients and 34310 non-HCC patients. They examined previously established covariates using Cox regression including laboratory MELD, In(AFP), number of tumors, and tumor size; all were significant predictors of dropout. The authors then equated the dropout hazard based on these covariates to that of non-HCC patients based 
on MELD alone to derive an equivalent MELD score. An approximation of the difference in log baseline hazard rates for dropout between HCC patients and non-HCC was made to determine a constant for less than 6 months after listing and another for waitlist time of 6 months or greater. Also, if the laboratory MELD was greater than the calculated MELD $D_{E Q}$, the MELD $D_{E Q}$ was taken to be the laboratory MELD. In addition, projections were made using multistate modeling to determine what transplant and dropout probabilities would be for HCC patients if the MELDEQ were implemented. This projection method is the same as that described in the study in Chapter 3 of this thesis, and is discussed there in detail. The authors of the original MELD $D_{\mathrm{EQ}}$ study found a concordance index of 0.741 for waitlist dropout. Advantages to the MELD $D_{E Q}$ include that it uses $\mathrm{HCC}$ characteristics as well as laboratory MELD and is on the same scale as the laboratory MELD. Also, projections for its use, stratified by ranges of the MELD and MELD $D_{E Q}$ scores, were made in its original publication. With these projections, the authors noted good alignment with the non-HCC groups in projected transplant probabilities, such that $\mathrm{HCC}$ patients and non-HCC patients with similar dropout risk based on MELD $\mathrm{EQ}_{\mathrm{Q}}$ and MELD strata also had similar projected transplant probabilities. A disadvantage, based on their projections, is that HCC projected dropout probabilities did not match the corresponding actual non-HCC probabilities as well as ideal for most strata; they were projected to decrease below non-HCC probabilities for higher risk strata. The investigators also noted that patients at higher risk for dropout as determined by their higher MELD $D_{E Q}$ score also had worse post-transplant survival, so a potential problem 
with this score could be increased transplantation in patients who may have poorer outcomes.

In summary, all of the proposed scores reviewed here have potential to improve equity between transplant and dropout probabilities between HCC and non-HCC patients, since they take HCC characteristics into account, and several of them took calculated HCC dropout risk into account. In contrast, the scheduled exception point progression that has been in place, and will still be in place after six months on the waiting list, assigns the same priority scores to all $\mathrm{HCC}$ patients regardless of their actual risk of dropout. Of the equivalent scores described here, the MELD $D_{E Q}$ and mixed new deMELD were derived most similarly, use similar covariates, and both are on the same scale as the MELD itself.

The primary aims of this thesis were to project outcomes for both HCC and non-HCC patients under the recently implemented six-month delay compared with under the MELD $D_{E Q}$, as well as evaluate alternative MELD scores for use by HCC patients. Evaluation of the six-month delay compared to the $M E L D_{E Q}$ will be discussed in Chapter 2. Since the MELD $D_{E Q}$ and the mixed new deMELD are the most similar as described above, they were selected for comparison with each other in terms of projected transplant and dropout probabilities. This comparison will be presented in Chapter 3. Concluding remarks and future research are given in Chapter 4.

Per the University of Louisville Human Subjects Protection Program Guide-006, the OPTN data used here are considered public and do not meet the definition of "human subject", so IRB review and approval was not needed. The 
data reported here have been supplied by UNOS as the contractor for OPTN.

The interpretation and reporting of these data are the responsibility of the author(s) and in no way should be seen as an official policy of or interpretation by the OPTN or the US Government. 


\section{CHAPTER 2}

\section{SIX-MONTH DELAY AND MELD ${ }_{E Q}$ COMPARISON}

As discussed in Chapter 1, under recent OPTN policies where exception points were given at listing and then progressed every three months for liver transplant candidates with $\mathrm{HCC}$, patients with these exceptions have had higher transplant rates and lower dropout rates compared to patients without $\mathrm{HCC}^{5,8}$. To address this, a delay of six months before granting exception points to HCC patients has recently been implemented. A secondary aim of this delay was to avoid transplanting HCC patients with aggressive tumors who may have a high recurrence risk post-transplant. Under the delay, candidates will be listed at their laboratory MELD scores until the second three month extension, at which time they will be assigned 28 points and continue with the scheduled progression of exception points. These exception points will be capped at $34^{3}$.

Other methods for addressing this disparity between $\mathrm{HCC}$ and non-HCC patients have been proposed, including equivalent MELD scores for HCC patients ${ }^{6,11-15}$. These scores were meant to reflect mortality risk for HCC patients more accurately than scheduled progression of exception points. These are described in Chapter 1, including the $M E L D_{E Q}$, which again was derived by determining dropout hazard rates based on established HCC characteristics as well as laboratory MELD and then equating this hazard to that of non-HCC patients to find the corresponding equivalent MELD score. Incidentally, as noted 
in Chapter 1, the MELD ${ }_{E Q}$ also included a waitlist time factor derived as part of the scoring system which increased an HCC patient's score after 6 months on the waiting list ${ }^{15}$. As it is on the same scale as the laboratory MELD score for non-HCC patients, it could be used comparably.

The main objective of this study was to compare the projected effect of the six-month delay to prioritization using $M E L D_{\mathrm{EQ}}$ scores on $\mathrm{HCC}$ and non-HCC dropout and transplant probabilities. These projections for HCC patients were studied for the MELD $D_{E Q}$ in its original publication ${ }^{15}$, and projections for transplant rates and mortality under the delay were previously studied by Heimbach et al using simulation methods ${ }^{10}$. However, the current study compares outcomes under both approaches and utilizes more recent UNOS data. Further, while the previous MELD $D_{E Q}$ study only evaluated projected effects on HCC patients, the current study includes projections for non-HCC patients as well. And though the earlier study by Heimbach et al demonstrated improved equity between $\mathrm{HCC}$ and non-HCC patients using the six-month delay, the effects of the delay on patients in various dropout strata were not specifically examined. Using UNOS data and multistate modeling, projected waitlist dropout and transplant probabilities were evaluated for both $\mathrm{HCC}$ and non-HCC patients in varying risk strata under the six-month delay and the MELD $D_{E Q}$ scoring system.

\section{METHODS}

\section{Data}

Data were obtained on all patients who were added to the UNOS liver transplant waitlist on or after 10/01/2009, based on OPTN data as of $06 / 30 / 14$, 
and who were at least 18 years old at time of initial listing. Patients with exceptions other than $\mathrm{HCC}$ were excluded, and the non-HCC dataset restricted to patients with no exceptions. Patients listed as status 1A or 1B and HCC patients who were missing HCC-related covariate data were also excluded.

\section{Outcomes and Covariates}

The main outcomes studied included actual and projected dropout and transplant probabilities for $\mathrm{HCC}$ and non-HCC patients based on the recent practice of scheduled progression of exception points, the six-month delay, and prioritization using MELD $D_{E Q}$ scores. The covariates used in the MELD $D_{E Q}$ model were the natural log of AFP (In(AFP)), laboratory MELD, maximum tumor size, and number of tumors. Dropout was defined as removal from the waiting list due to death, determined medically unsuitable, or too sick for transplant. Transplant was defined as having received transplant for any reason. Those who remained on the waiting list or were removed due to improvement were considered censored.

\section{Statistical Methods}

The MELD $D_{E Q}$ was calculated for all HCC patients at each follow-up time using the following equation:

$$
\begin{aligned}
\text { MELD }_{\mathrm{EQ}}= & \max \left(\text { lab MELD, } 1.143^{*} \mathrm{MELD}+1.324^{*} \ln (\text { AFP })\right. \\
& \left.+1.438^{*} \text { Number of Tumors }+1.194^{*} \text { Max Tumor Size }+\mathrm{c}(\mathrm{t})\right) \\
& \text { where } \mathrm{c}(\mathrm{t})=-2 / 0.146 \text { for } \mathrm{t}<6 \text { months } \\
& \text { and } \mathrm{c}(\mathrm{t})=-1 / 0.146 \text { for } \mathrm{t} \geq 6 \text { months }
\end{aligned}
$$


Observations were categorized into ranges based on their MELD $D_{E Q}$ and lab MELD scores. These ranges included <12, 12-15, 16-21, 22-27, and 28-40. Scores of 22 and above were categorized based on the previous exception points granted at three-month intervals and then combined due to sparse data in the higher risk groups.

These categories were used as the transient states in a nonparametric multistate model for dropout and transplant probabilities, using the $\mathrm{R}$ package msSurv ${ }^{21}$. Figure 1 displays a schematic diagram of the multistate model used for this portion of the study. In a multistate model, patients can transition between the transient states at any time but cannot transition out of the terminal states. Briefly, our multistate model consists of five transient states (the MELD / $M_{E L D}$ risk categories, labeled states 1-5) and two terminal states of waitlist dropout (state 6) and transplantation (state 7). Let the number of patients in state $i$ at time $t$ be $Y_{i}(t)$. These individuals are the number 'at risk' of making a transition out of state $i$ at that time. The number of individuals making a transition from state $i$ to state $j$ at time $t$ is denoted $d N_{i j}(t)$. Then, the nonparametric estimate of the transition hazard from state $i$ to state $j$ at time $t$ is $d \hat{A}_{i j}(t)=d N_{i j}(t) / Y_{i}(t)$. These rates give the instantaneous risk of transition from state $i$ to state $j$ at that time. These transition hazards are combined into the state transition intensity matrix $d \widehat{\mathbf{A}}(t)$ (a $7 \times 7$ matrix in this case), with the offdiagonal elements given by the $d \hat{A}_{i j}(t)$ terms and the diagonal elements equal to $d \hat{A}_{i i}(t)=-\sum_{j \neq i} d \hat{A}_{i j}(t)$. The state transition intensity matrices are then used to estimate the state transition probability matrix $\mathbf{P}(s, t)$, where each element in the 
matrix $P_{i j}(s, t)$ gives the probability of making a transition starting from state $i$ at time $s$ to state $j$ at time $t$. The elements of $\mathbf{P}(s, t)$ are estimated by the AalenJohansen estimator $^{22} \widehat{\mathbf{P}}(s, t)=\prod_{u \in(s, t]}(\mathbf{I}+d \widehat{\mathbf{A}}(u))$, where $u$ indicates the event times and I denotes the identity matrix (in this case a $7 \times 7$ matrix). The multistate model was used to calculate probabilities for transplant and waitlist dropout (terminal states) for each risk category (transient states). The model accounts for transitioning between these states prior to dropout or transplant, as well as transitioning directly to the terminal states.

Dropout and transplant probabilities were modeled for HCC patients. One model was fitted for HCC patients stratified by laboratory MELD ranges for evaluation of the six-month delay and another with stratification using MELD $D_{E Q}$. A similar model was constructed for non-HCC patients. Actual dropout and transplant probabilities were obtained from these models.

To address the possible concern of early transplant of high-risk HCC patients who may have worse post-transplant outcomes, we compared posttransplant survival between high-risk $\mathrm{HCC}$ patients $\left(M E L D_{\mathrm{EQ}} \geq 28\right)$ transplanted within six months from listing and those transplanted after six months. KaplanMeier survival estimates and the log-rank test were used for this comparison.

All data analysis and statistical calculations were performed using SAS (Version 9.4, Cary, NC) and R for Windows (Version 3.2.2).

\section{Projected Outcomes}

The projection estimates assume that $\mathrm{HCC}$ and non-HCC patients with the same MELD / equivalent MELD strata are transplanted at the same rate. This is similar to the projection method described in the original MELD $\mathrm{EQ}_{\mathrm{EQ}}$ paper $^{15}$, 
however here we modify the approach to account for the potential impact on nonHCC patients as well. This is done by assuming that the total number of available organs remains fixed, and organs are redistributed across strata according to non-HCC rates. The technical details for the projection method are given below.

Projected transplant hazard rates were obtained based on current non$\mathrm{HCC}$ transplant rates for each MELD / MELD $\mathrm{EQ}_{\mathrm{E}}$ stratum $i, i=1, \ldots, 5$, in the following manner (recall that the strata are defined as MELD / MELD ${ }_{E Q}$ score ranges of $<12,12-15,16-21,22-27$, and 28-40). Let $d N_{i 7}^{\mathrm{HCC}}(t)$ be the total number of transplants to HCC patients in stratum $i$ at time $t$ (here $t=$ time since listing in our multi-state model) and let $d N_{i 7}^{\mathrm{nHCC}}(t)$ be analogously defined for non-HCC patients (recall that state 7 is the transplantation state in our model, see Figure 1). For non-HCC patients being transplanted at time $t$, the conditional probability that the organ goes to the $i$ th strata is $p_{i}(t)=d N_{i 7}^{\mathrm{nHCC}}(t) / \sum_{k=1}^{5} d N_{k 7}^{\mathrm{nHCC}}(t)$. In the projections, these conditional probabilities for non-HCC patients are used to determine the probability that a transplanted organ at time $t$ goes to the $i$ th strata. Let the total number of organs (combined $\mathrm{HCC}$ and non-HCC) being transplanted at time $t$ be $d N_{\cdot 7}(t)=$ $\sum_{k=1}^{5}\left(d N_{k 7}^{\mathrm{nHCC}}(t)+d N_{k 7}^{\mathrm{HCC}}(t)\right)$. Then the redistributed number of organs going to each strata ( $\mathrm{HCC}$ and non-HCC combined) at time $t$ are defined as $d \widetilde{N}_{i 7}(t)=$ $d N_{.7}(t) p_{i}(t)$. The fractions of these going to $\mathrm{HCC}$ and non-HCC patients are determined by the relative number at risk in each group at that time. That is, the projected number of organs going to $\mathrm{HCC}$ patients in stratum $i$ at time $t$ is 
$d \widetilde{N}_{i 7}^{\mathrm{HCC}}(t)=\left(\frac{Y_{i}^{\mathrm{HCC}}(t)}{Y_{i}^{\mathrm{HCC}}(t)+Y_{i}^{\mathrm{HCC}}(t)}\right) d \widetilde{N}_{i 7}(t)$, where $Y_{i}^{\mathrm{HCC}}(t)$ is the number of HCC patients in stratum $i$ just prior to time $t$ and $Y_{i}^{\mathrm{nHCC}}(t)$ is analogously defined for non-HCC patients. Similarly, for non-HCC patients the projected number would be $d \widetilde{N}_{i 7}^{\mathrm{nHCC}}(t)=\left(\frac{Y_{i}^{\mathrm{nHCC}}(t)}{Y_{i}^{\mathrm{HCC}}(t)+Y_{i}^{\mathrm{nHCC}}(t)}\right) d \widetilde{N}_{i 7}(t)$.

Once the projected number of transplanted organs are calculated for both $\mathrm{HCC}$ and non-HCC patients for every stratum $i$ and time $t$, projected transplant rates can be calculated as $d \tilde{A}_{i 7}^{\mathrm{HCC}}(t)=d \widetilde{N}_{i 7}^{\mathrm{HCC}}(t) / Y_{i}^{\mathrm{HCC}}(t)$ and $d \tilde{A}_{i 7}^{\mathrm{nHCC}}(t)=$ $d \widetilde{N}_{i 7}^{\mathrm{nHCC}}(t) / Y_{i}^{\mathrm{nHCC}}(t)$. These transplant rates are substituted into the estimated state transition intensity matrix $d \widehat{\mathbf{A}}(t)$ defined previously. Denote these matrices with the substituted projected transplant rates for $\mathrm{HCC}$ and non-HCC patients as $d \widetilde{\boldsymbol{A}}^{\mathrm{HCC}}(t)$ and $d \widetilde{\boldsymbol{A}}^{\mathrm{nHCC}}(t)$, respectively. Diagonal elements of $\mathbf{I}+d \widetilde{\boldsymbol{A}}(t)$ are adjusted accordingly so that each row sums to one. Then the projected transplant and dropout probabilities can be calculated using the Aalen-Johansen estimator with these newly defined transition intensity matrices as $\widetilde{\boldsymbol{P}}^{\mathrm{HCC}}(s, t)=$ $\prod_{u \in(s, t]}\left(\mathbf{I}+d \widetilde{\boldsymbol{A}}^{\mathrm{HCC}}(u)\right)$ and $\widetilde{\boldsymbol{P}}^{\mathrm{nHCC}}(s, t)=\prod_{u \in(s, t]}\left(\mathbf{I}+d \widetilde{\boldsymbol{A}}^{\mathrm{nHCC}}(u)\right)$.

The projected transplant hazards were calculated as above for both the six-month delay model and the MELD $\mathrm{EQ}_{\mathrm{EQ}}$ model. For projections under the sixmonth delay, use of the actual transplant hazards in the Aalen-Johansen estimator were resumed after six months to reflect reverting to scheduled exception point progression. For projections under the $M E L D_{E Q}$, the probabilities were calculated using projected transplant hazards through 18 months.

To project outcomes for patients under the MELDEQ given a patient is still 
on the list after 6 months, separate calculations were made for $6-18$ months from listing. Calculations were made in an analogous fashion using the non-HCC multistate model, the MELD $D_{\mathrm{EQ}} \mathrm{HCC}$ multistate model, and projected transplant hazards as described in the previous paragraphs. However, transition times starting at six months from listing were used.

Overall projected and actual dropout and transplant probabilities at six, 12 and 18 months since time of listing were calculated using the multistate models for the six-month delay, the MELD $D_{E Q}$ and the prioritization scheme in place during the time of the study. The variances of these estimates for actual probabilities were obtained using the bootstrap option in the $\mathrm{R}$ package $m s S u r v^{21}$, with 200 iterations, and used to construct normal 95\% confidence intervals. For projected overall probabilities, 500 bootstrap iterations of the projected estimates were performed and checked graphically for normality. Then their standard deviations were used to construct normal 95\% confidence intervals. 
Figure 1: Schematic Diagram of Multistate Model. Transient states consisting of MELD or MELD $D_{E Q}$ ranges are represented by circles, while the terminal states of dropout and transplant are represented by rectangles. Transitions are possible between any of the transient states (light lines), and from any transient state to the terminal states (bold lines). Transitions to the dropout / transplant states are of primary interest.

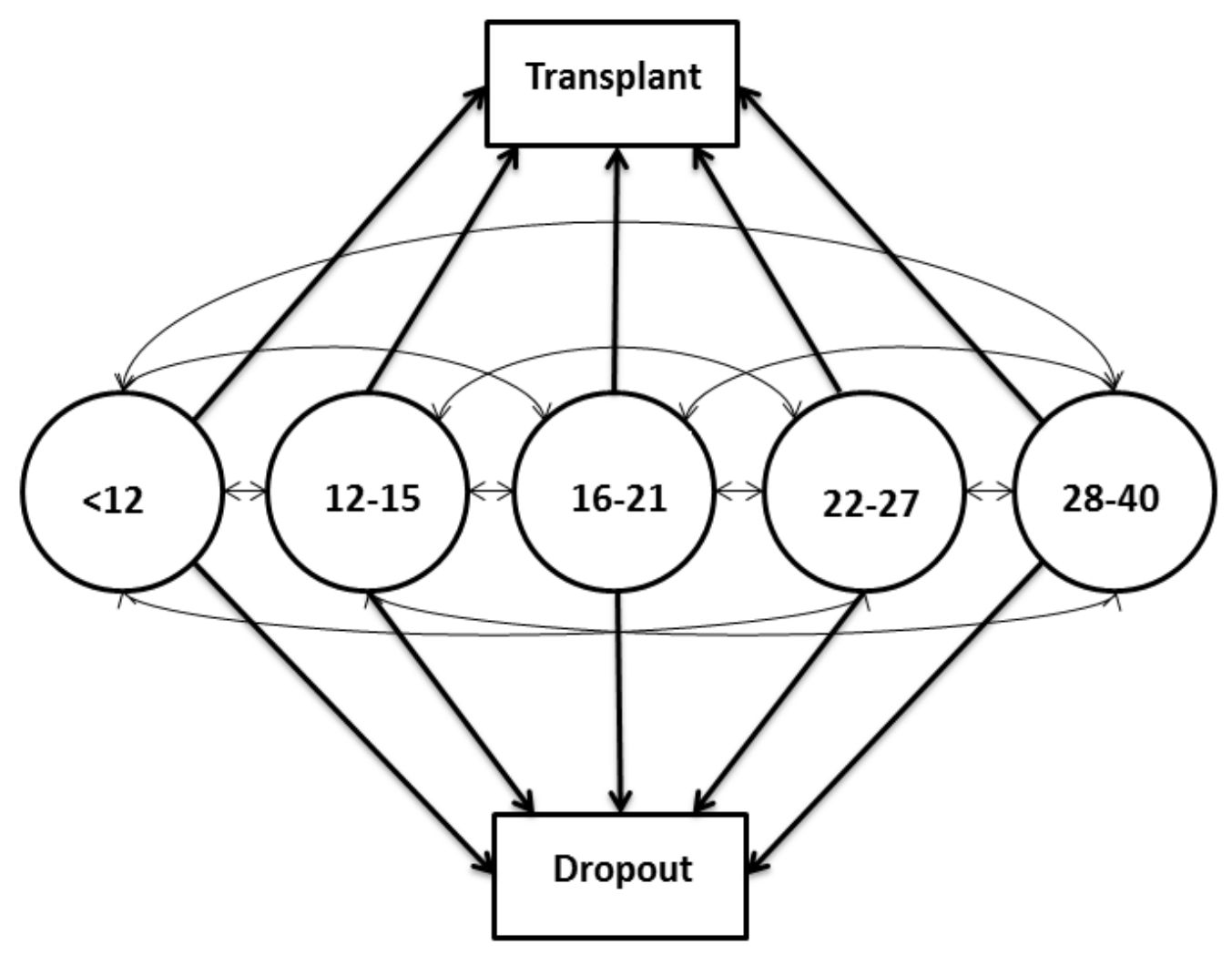




\section{RESULTS}

A total of 7,931 patients were listed with HCC exceptions and 34,868

patients with standard MELD scores during the time frame between 10/1/2009 and 6/30/2014. Three HCC patients were missing HCC-related covariate data, leaving 7,928 for analysis. Table 1 shows the numbers and percentages of HCC and non-HCC patients who were censored, dropped out, received transplant, or improved as of last follow-up. Compared to non-HCC, HCC patients had a higher percentage of transplantation ( $71 \%$ vs. $39.9 \%)$ and a lower percentage of dropout $(5.7 \%$ vs. $10.6 \%)$.

\begin{tabular}{l|l|l} 
& HCC & $\begin{array}{l}\text { Non-HCC } \\
\text { Outcome }\end{array}$ \\
Count (percent) & Count (percent) \\
\hline Censored & $1837(23.2 \%)$ & $16633(47.7 \%)$ \\
Dropout & $452(5.7 \%)$ & $3700(10.6 \%)$ \\
Transplanted & $5629(71 \%)$ & $13895(39.9 \%)$ \\
Improved & $10(0.1 \%)$ & $640(1.8 \%)$ \\
Total & 7928 & 34868
\end{tabular}

Table 1: Outcomes at last follow up for $\mathrm{HCC}$ and non-HCC patients. Numbers in each cell are the count and percentage of patients out of 7928 total HCC patients and 34868 non-HCC patients.

Figure 2 displays actual and projected dropout and transplant probabilities for patients stratified by their laboratory MELD ranges during 0-18 months from listing. As expected, the transplant probabilities under recent practice are similar for HCC patients regardless of MELD score range (Figure 2a). There is some differentiation in actual dropout probabilities for HCC patients stratified by 
laboratory MELD, though not as clear as that for non-HCC patients (Figure 2c). The separation in projected transplant probabilities by dropout risk strata under the six-month delay is much more distinct than before, though lower than the corresponding non-HCC patients in the same ranges (Figure $\mathbf{2 b}$ ) for the first six months. We see an increase in projected transplant probabilities compared to actual for HCC patients in strata with MELD scores $>21$ for the first 6 months, but a decrease for those in lower strata (c.f. Figure 2a vs. 2b). However, after six months, projected transplant probabilities rapidly increase for HCC patients to again exceed those for non-HCC patients (Figure 2b). Under the delay, dropout probabilities for HCC patients with MELD scores $<22$ are projected to be slightly higher compared to current levels (c.f. Figure $\mathbf{2 d}$ vs. $\mathbf{2 c}$ ) though patients with higher MELD scores are expected to have reduced dropout probabilities relative to current. Projected dropout probabilities are also somewhat higher for HCC patients with MELD scores $<22$ relative to non-HCC patients, but lower for HCC than non-HCC for patients with MELD scores $\geq 22$. For non-HCC patients, dropout probabilities are projected to be slightly reduced for those with MELD $>15$ (c.f. Figure 2c vs. 2 d).

Projections under MELD $D_{E Q}$ are very similar to those for the six-month delay for the first six months of listing, as seen by comparing Figures $\mathbf{2}$ and $\mathbf{3}$ for the first six months shown. During these first six months, the MELD $D_{E Q}$ score is equivalent to the laboratory MELD score for $93 \%$ of observations. Table 2 shows the distributions of the MELD $D_{E Q}$ and laboratory MELD for HCC patients at listing. 


\begin{tabular}{l|l|l} 
Score Ranges & $\begin{array}{l}\text { HCC MELD } \\
\text { Count (percent) }\end{array}$ & $\begin{array}{l}\text { HCC Lab MELD } \\
\text { Count (percent) }\end{array}$ \\
\hline $6-11$ & $4759(60.0 \%)$ & $4889(61.7 \%)$ \\
$12-15$ & $2075(26.2 \%)$ & $2017(25.4 \%)$ \\
$16-21$ & $959(12.1 \%)$ & $911(11.5 \%)$ \\
$22-27$ & $126(1.6 \%)$ & $102(1.3 \%)$ \\
$28-40$ & $9(0.1 \%)$ & $9(0.1 \%)$
\end{tabular}

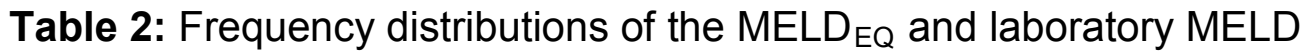
scores for HCC patients at initial listing. Numbers in each cell are the count and percentage of patients out of 7928 total HCC patients.

Figure 3 shows actual and projected dropout and transplant probabilities under the MELDEQ scoring method through 18 months from listing. At six months, a slight increase in projected HCC transplant probabilities is seen under this scheme similarly to the six-month delay, but the increase is much less pronounced. This increase would be expected as the MELD ${ }_{E Q}$ assigns 6.85 more points when a patient has been on the waiting list for at least six months.

As with laboratory MELD, actual transplant probabilities are similar over time for all HCC patients regardless of risk strata as determined by the MELD $D_{E Q}$ (Figure 3a). Under the MELD $D_{\mathrm{EQ}}$ model, projected transplant probabilities are much more defined according to risk strata (Figure 3b), and thus closer to nonHCC probabilities compared to actual transplant probabilities for most strata. In contrast to Figure $\mathbf{2 b}$, they remain this way after six months on the waitlist. However, they are less than projected non-HCC probabilities in the corresponding strata. Non-HCC transplant probabilities are projected to increase slightly for all strata under this model (c.f. Figure 3a vs. 3b), while non-HCC 
dropout would decrease slightly for all but the lowest stratum (c.f. Figure $3 \mathrm{c}$ vs 3d). Under the $M E L D_{E Q}$, dropout probabilities are projected to increase for those with scores $<22$ and decrease for those with scores $\geq 22$ (Figures $3 \mathrm{c}$ and $\mathbf{3 d}$ ).

Figure 4 shows actual and projected transplant and dropout probabilities for HCC patients stratified by MELD $\mathrm{EQ}_{\mathrm{S}}$ scores and non-HCC by MELD scores for six months through 18 months after listing. These curves reflect the probability of transplant or dropout for a patient given she/he is still on the waitlist at six months. During this time frame, actual transplant probabilities (shown in Figure 4a) for HCC patients exceed those for non-HCC patients except for the highest non-HCC risk group (MELD $\geq 28$ ). Projected transplant probabilities under the $M E L D_{E Q}$ match those for non-HCC patients in corresponding ranges well (Figure 4b). Projected dropout probabilities for HCC patients under this scheme exceed the levels for the corresponding non-HCC strata (Figure 4d). Compared with actual dropout (Figure 4c), projected probabilities would again be reduced for HCC patients with MELD $D_{E Q}$ scores $\geq 22$ but increased for those with scores $<22$. For non-HCC patients, projected dropout probabilities are similar to actual for MELD $<16$ and reduced for MELD $\geq 16$. The projections on Figure 4 reflect dropout and transplant probabilities under the MELD $D_{E Q}$ as if it were used beginning at six months waitlist time. The results displayed in Figures $\mathbf{2}$ and $\mathbf{3}$ are aggregated and summarized in Table 3, which gives overall dropout and transplant probabilities and their 95\% confidence intervals under recent practice ("actual" on Figure 3) and projected for the six-month delay and the MELD $D_{E Q}$ at 6, 12 and 18 months after listing. Under the six-month delay, overall dropout at 6 months is projected to increase slightly for HCC patients and decrease slightly for 
non-HCC patients compared to actual probabilities, as expected, while overall transplant probabilities would be reduced for HCC patients and increased for non-HCC patients. As noted previously, these results are very similar for the $M E L D_{E Q}$ during the first six months on the waitlist.

At 12 months waitlist time, the overall dropout probability would increase somewhat for $\mathrm{HCC}$ patients under both the six-month delay and the MELDEQ, while it would decrease for non-HCC patients. This change is slightly greater under the MELD $D_{E Q}$. Again, the opposite change would be seen for transplant probabilities (increase for non-HCC and decrease for $\mathrm{HCC}$ ) under these schemes. This change is more pronounced under the MELD $D_{\mathrm{EQ}}$. The same trend is seen at 18 months waitlist time. For all of these probabilities, the point estimates and 95\% confidence intervals are shown in Table 3.

Table 3c shows actual and projected dropout probabilities for total HCC and non-HCC patients, weighted by the proportion of each group to the whole sample. Both the six-month delay and the $M E L D_{E Q}$ are projected to decrease total dropout probabilities compared to actual, though in neither case is the result statistically significant as indicated by the overlap in $95 \%$ confidence intervals (Cls). While the total projected dropout is nearly identical between the MELD $D_{E Q}$ and the six-month delay at the various time points, the probabilities become closer over time between HCC and non-HCC patients under the MELD $D_{\mathrm{EQ}}$ (see Table 3a and 3b). At 12 months, projected dropout probabilities under the MELD $D_{E Q}$ are $8.38 \%$ and $8.89 \%$ for $\mathrm{HCC}$ and non-HCC respectively, and at 18 months they are $10.82 \%$ and $10.22 \%$. Under the six-month delay, overall 
projected dropout for non-HCC patients is still statistically significantly higher than that for HCC patients at these time points (95\% Cls do not overlap).

\begin{tabular}{|l|c|c|c|c|c|c|}
\cline { 2 - 7 } \multicolumn{1}{c|}{} & \multicolumn{5}{c|}{ a. HCC Patients } \\
\cline { 2 - 7 } \multicolumn{1}{c|}{} & \multicolumn{3}{c|}{ Dropout } & \multicolumn{3}{c|}{ Transplant } \\
\cline { 2 - 7 } \multicolumn{1}{c|}{} & Actual & $\begin{array}{c}\text { 6-Month } \\
\text { Delay }\end{array}$ & MELD $_{\mathrm{EQ}}$ & Actual & $\begin{array}{c}\text { 6-Month } \\
\text { Delay }\end{array}$ & MELD $_{\mathrm{EQ}}$ \\
\hline $\mathbf{6}$ & $3.68 \%$ & $4.58 \%$ & $4.54 \%$ & $43.33 \%$ & $8.18 \%$ & $8.54 \%$ \\
Mo & $3.25,4.11$ & $4.02,5.15$ & $3.98,5.1$ & $42.21,44.45$ & $7.76,8.61$ & $8.11,8.97$ \\
\hline $\mathbf{1 2}$ & $5.63 \%$ & $7.73 \%$ & $8.38 \%$ & $66.29 \%$ & $45.92 \%$ & $16.5 \%$ \\
Mo & $5.11,6.15$ & $6.94,8.52$ & $7.49,9.28$ & $65.18,67.4$ & $44.33,47.52$ & $15.82,17.17$ \\
\hline $\mathbf{1 8}$ & $6.42 \%$ & $9.02 \%$ & $10.82 \%$ & $80.97 \%$ & $70.2 \%$ & $21.63 \%$ \\
Mo & $5.85,6.99$ & $8.13,9.91$ & $9.63,12.01$ & $79.92,81.95$ & $68.53,71.88$ & $20.73,22.53$ \\
\hline
\end{tabular}

\begin{tabular}{|l|c|c|c|c|c|c|}
\cline { 2 - 7 } \multicolumn{1}{c|}{} & \multicolumn{6}{c|}{ b. Non-HCC Patients } \\
\cline { 2 - 7 } \multicolumn{1}{c|}{} & \multicolumn{5}{c|}{ Dropout } & \multicolumn{3}{c|}{ Transplant } \\
\cline { 2 - 7 } \multicolumn{1}{c|}{} & Actual & $\begin{array}{c}\text { 6-Month } \\
\text { Delay }\end{array}$ & MELD $_{\text {EQ }}$ & Actual & $\begin{array}{c}\text { 6-Month } \\
\text { Delay }\end{array}$ & MELD $_{\text {EQ }}$ \\
\hline $\mathbf{6}$ & $7.71 \%$ & $7.01 \%$ & $7.01 \%$ & $35.95 \%$ & $41.19 \%$ & $41.14 \%$ \\
Mo & $7.41,8.01$ & $6.74,7.27$ & $6.75,7.28$ & $35.45,36.45$ & $40.66,41.72$ & $40.62,41.67$ \\
\hline $\mathbf{1 2}$ & $10.15 \%$ & $9.1 \%$ & $8.89 \%$ & $42.74 \%$ & $46.84 \%$ & $49.77 \%$ \\
Mo & $9.78,10.52$ & $8.79,9.42$ & $8.59,9.2$ & $42.17,43.31$ & $46.31,47.37$ & $49.25,50.29$ \\
\hline $\mathbf{1 8}$ & $11.9 \%$ & $10.7 \%$ & $10.22 \%$ & $46.4 \%$ & $50.1 \%$ & $54.49 \%$ \\
Mo & $11.51,12.29$ & $10.34,11.05$ & $9.89,10.56$ & $45.76,47.04$ & $49.56,50.65$ & $53.96,55.02$ \\
\hline
\end{tabular}

\begin{tabular}{|l|c|c|c|}
\cline { 2 - 4 } \multicolumn{1}{c|}{} & \multicolumn{3}{c|}{ C. Total (HCC and non-HCC) Dropout } \\
\cline { 2 - 4 } \multicolumn{1}{c|}{} & Actual & 6-Month Delay & MELD $_{\text {EQ }}$ \\
\hline $\mathbf{6}$ & $6.96 \%$ & $6.56 \%$ & $6.55 \%$ \\
Mo & $6.64,7.29$ & $6.24,6.88$ & $6.24,6.87$ \\
\hline $\mathbf{1 2}$ & $9.31 \%$ & $8.85 \%$ & $8.8 \%$ \\
Mo & $8.92,9.71$ & $8.45,9.25$ & $8.39,9.21$ \\
\hline $\mathbf{1 8}$ & $10.88 \%$ & $10.39 \%$ & $10.33 \%$ \\
Mo & $10.46,11.31$ & $9.93,10.84$ & $9.84,10.83$ \\
\hline
\end{tabular}

Table 3: Overall dropout/transplant probabilities with $95 \%$ confidence intervals for $\mathrm{HCC}$ and non-HCC patients, actual and projected with prioritization under the 6-month delay and MELD $D_{\mathrm{EQ}}$ at 6,12 , and 18 months waitlist time. 

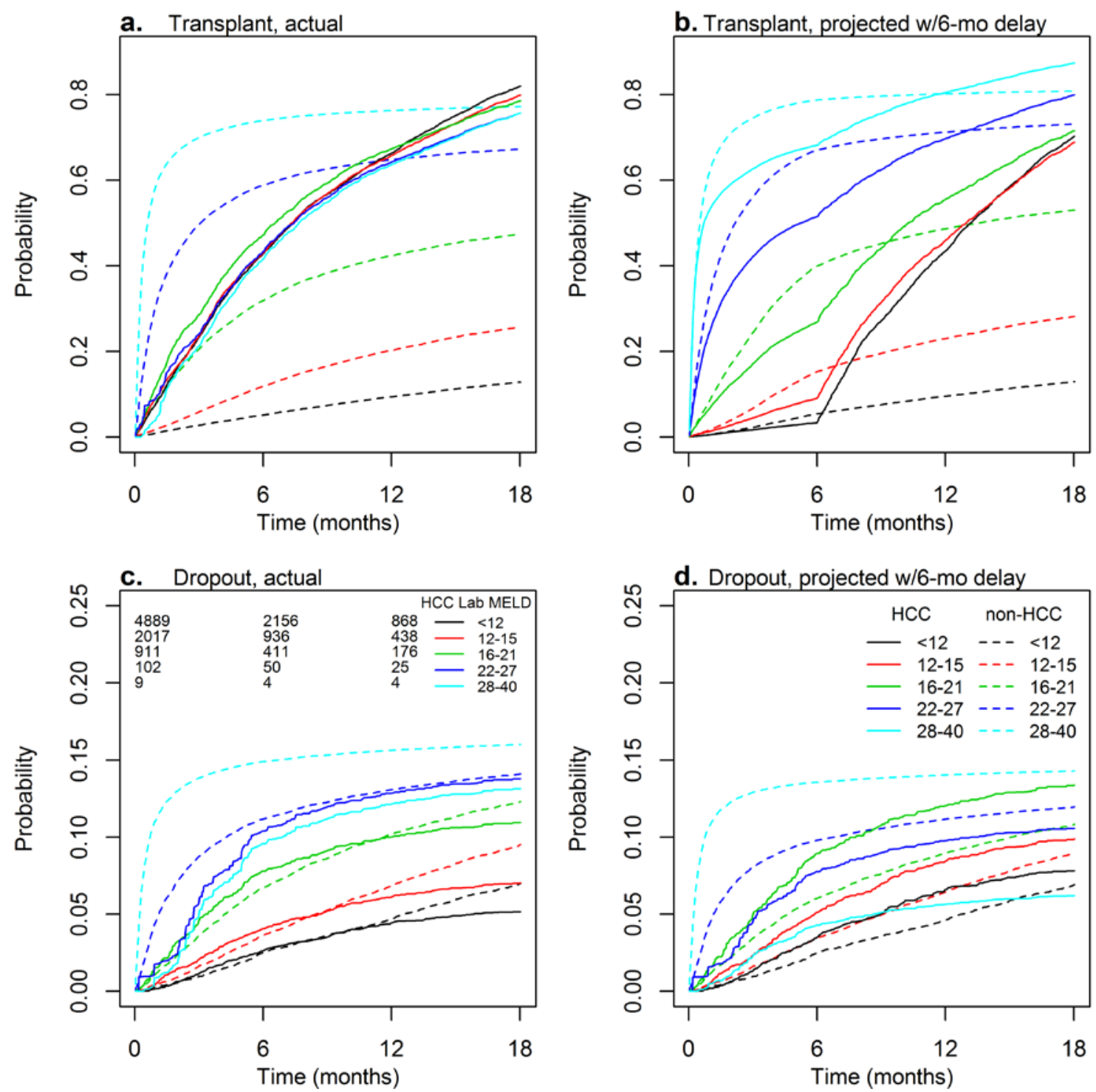

Figure 2: Actual and projected time to transplant and dropout for HCC and non-HCC patients stratified by laboratory MELD score. Time is from 0 to 18 months after listing. Solid lines indicate the probability of transplant/dropout for HCC patients under the current scheme $(a, c)$ and under the six-month delay in assigning exception points (b, d). Dashed lines indicate the corresponding probability curves for non-HCC patients. The number at risk for $\mathrm{HCC}$ patients at baseline, 6 , and 12 months is given in the upper part of panel $\mathrm{c}$. 

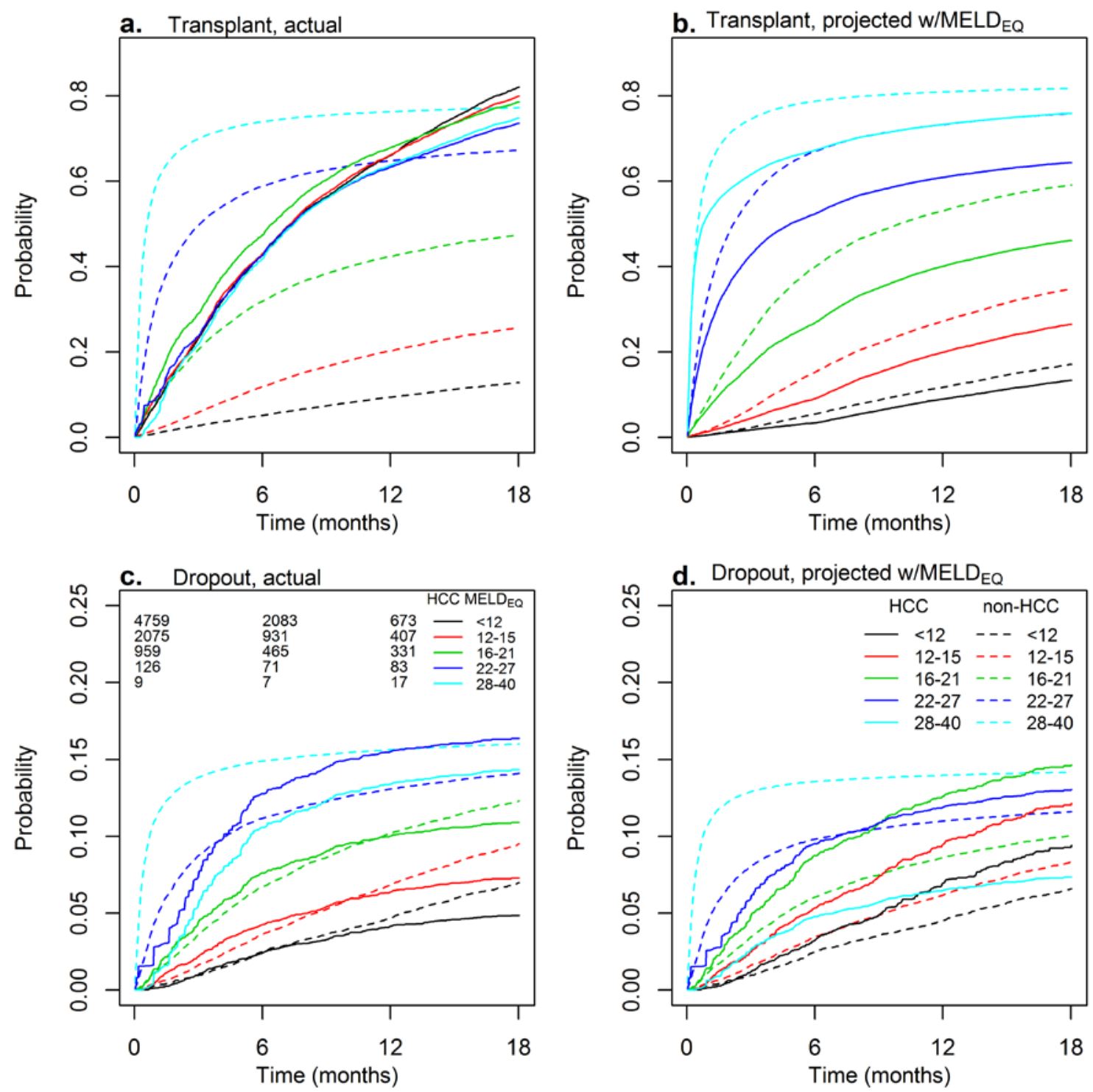

Figure 3: Actual and projected time to dropout or transplant for HCC patients stratified by MELD ${ }_{E Q}$ score and non-HCC patients by MELD. Time is from 0-18 months after listing. The curves reflect the probability of transplant or dropout under the current scheme $(\mathrm{a}, \mathrm{c})$ and those projected under the MELD $\mathrm{EQ}_{\mathrm{EQ}}$ scoring system (b, d). Solid lines represent probabilities for HCC patients while dashed lines represent those for non-HCC patients. The number at risk for $\mathrm{HCC}$ patients at baseline, 6 , and 12 months is given in the upper part of panel $\mathrm{c}$. 

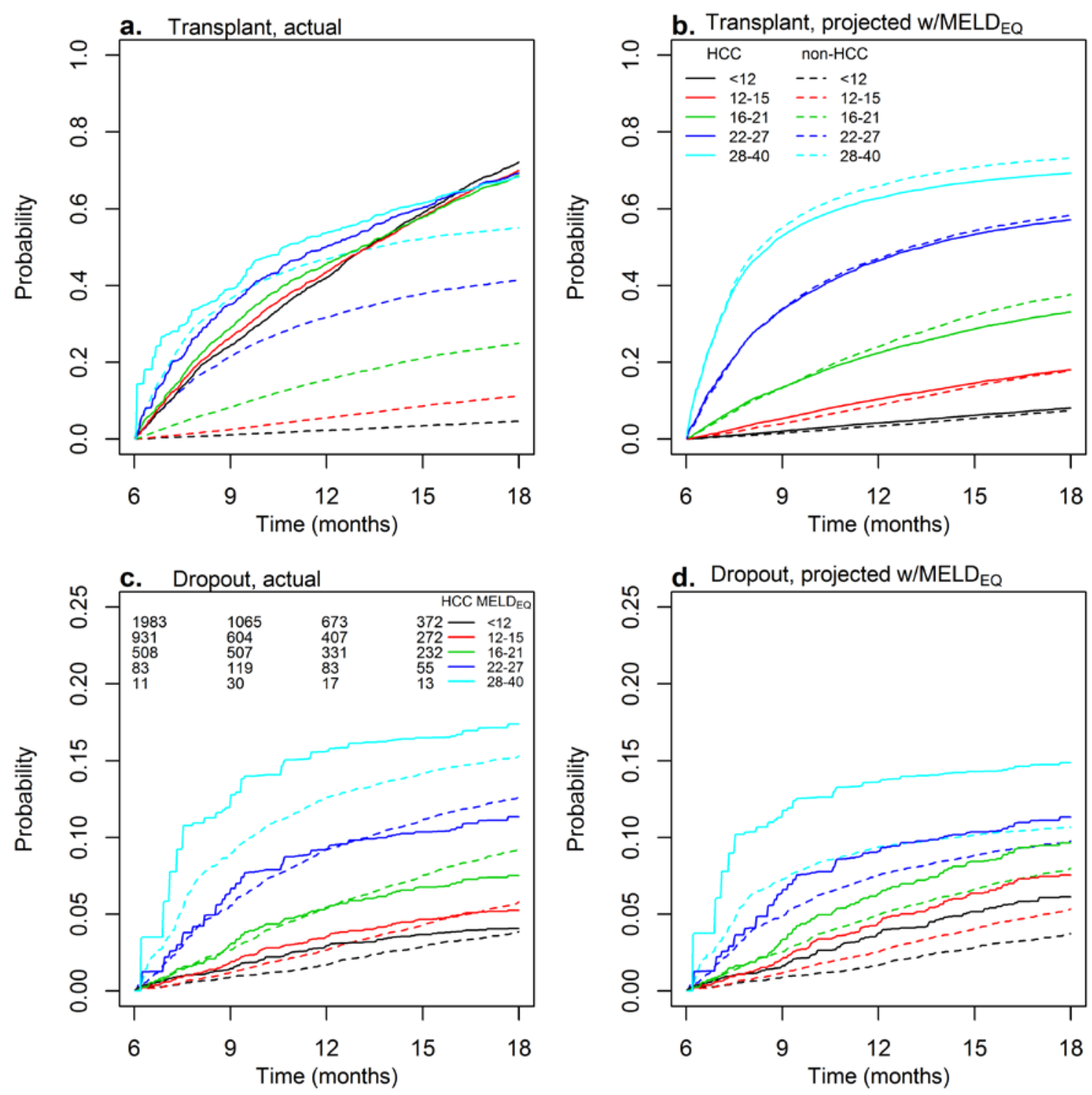

Figure 4: Actual and projected time to dropout or transplant for HCC patients stratified by MELD ${ }_{E Q}$ score and non-HCC patients by MELD. Time is from 6 months until 18 months after listing. The curves reflect the probability, given a patient is still on the waiting list at 6 months, of transplant or dropout under the current scheme $(a, c)$ and those projected under the $M E L D_{E Q}$ scoring system (b, d). Solid lines represent probabilities for HCC patients while dashed lines represent those for non-HCC patients. The number at risk for HCC patients at $6,9,12$, and 15 months is given in the upper part of panel $\mathrm{c}$. 
Lastly, since part of the rationale of the six month delay was to possibly avoid transplanting high-risk HCC patients earlier who may have poorer outcomes, post-transplant survival was compared between patients classified as higher-risk $\left(M E L D_{E Q} \geq 28\right)$ and transplanted within six months $(n=26)$ vs. after six months $(n=81)$ from listing. Although post-transplant survival was somewhat lower for those transplanted earlier, this was not statistically significant $(p=0.285)$.

\section{DISCUSSION}

In this part of the study, projections were evaluated for dropout and transplant probabilities under the recently implemented six-month delay for granting exception MELD points to HCC patients and compared to prioritization based on the MELD $D_{E Q}$. While the six-month delay will improve equity of transplant probabilities between $\mathrm{HCC}$ and non-HCC patients for the first six months on the waiting list, these results show that reverting to scheduled exception point progression after six months will again advantage $\mathrm{HCC}$ patients. Additionally, reverting to exception points after six months still treats all HCC patients as having the same dropout risk regardless of tumor characteristics or laboratory MELD.

Dropout was projected to increase under the delay for HCC patients with MELD scores <22, but projected to decrease for both HCC and non-HCC patients with MELD scores $\geq 22$. This increase in dropout for lower HCC risk groups is in contrast to findings in the original study describing the derivation of the MELD $D_{E Q}{ }^{15}$. In that study, it was projected that patients with MELD $D_{E Q}$ scores $\leq 15$ would not be adversely affected by the six-month delay while those with 
higher scores could be adversely affected in terms of dropout probability. It was noted that the advantage to $\mathrm{HCC}$ patients was not universal, as dropout probabilities for lower risk HCC patients $\left(M E L D_{E Q} \leq 15\right)$ were lower than their nonHCC counterparts while their transplant probabilities were higher. The opposite (higher dropout, lower transplant) was true for patients in higher $M E L D_{E Q}$ ranges. While this appears to still be the case for transplant probabilities, dropout for most HCC groups in the current study appears to be lower than in the original MELD $D_{E Q}$ study. Overall HCC dropout probabilities in the current study were found to be $3.68 \%$ at 6 months and $5.63 \%$ at 12 months while in the earlier study they were $4.7 \%$ at 6 months and $7.2 \%$ at 12 months ${ }^{15}$. Also, since the original $M E L D_{E Q}$ study modeled dropout and transplant since the time a patient first entered a score range and the MELDEQ assigns more points once a patient has been on the waitlist for six months, it is likely that some of the higher risk patients identified in the earlier study had already been on the list for six months or more. In order to make comparisons with the six-month delay, in this study we modeled these probabilities using time since listing.

While dropout probabilities were projected to increase somewhat for HCC patients under both the six-month delay and the MELD $D_{E Q}$, the magnitude of this increase was much smaller than that of the projected decrease in HCC transplant probabilities. This was particularly evident under the MELD $D_{E Q}$. Results from the paper by Heimbach et al also suggested that increased mortality rates may not accompany decreased transplant rates for HCC patients ${ }^{10}$. However, the reduction of $\mathrm{HCC}$ transplant probabilities below those of non-HCC candidates is still a limitation of the $M E L D_{E Q}$, as ideal prioritization would equalize both 
transplant and dropout probabilities between $\mathrm{HCC}$ and non-HCC patients overall and according to dropout risk strata.

Other investigators have looked at the balance between waiting time, waitlist dropout, post-transplant survival and equity of transplant rates, and found that increased waiting time was associated with either improved overall survival (while on the waiting list and post-transplant) or post-transplant survival ${ }^{23-25}$. The possible benefits and risks of increased waiting time were also discussed in a paper by Roayaie \& Roayaie, who point out that while increased waiting time was associated with longer post-transplant survival, waiting too long begins to remove patients who would have done well post-transplant ${ }^{26}$. While findings here include slightly lower post-transplant survival for HCC patients transplanted earlier versus later, this result was not statistically significant. It should be noted that this comparison was only made for patients with MELD $D_{E Q}$ of at least 28 at their last follow up prior to transplant, which was a small sample size $(n=107)$. Increased waiting time was not the primary goal of the MELD $D_{E Q}$, though it does assign a higher score once a candidate has been on the list for six months.

A limitation of this study is that the representation of organ availability used in the projections may not be completely realistic since projections were modeled using time since listing rather than calendar time. That is, the number of organs estimated to be available at a certain time $t$ is based on how many candidates received an organ at that time $t$ since listing. However, this method still provides an estimate of the proportion of transplants going to each group and the projected change for $\mathrm{HCC}$ relative to non-HCC candidates under each prioritization system. Also, evaluating three prioritization systems this way can 
still provide a valid comparison of them, as they were all compared using the same projection method.

Strengths of the study include examination of the effects of the six-month delay and the MELD $D_{E Q}$ over time, including projections after six months on the waitlist, and study of these effects among different HCC dropout risk strata. While equalizing $\mathrm{HCC}$ and non-HCC dropout and transplant probabilities overall is the primary goal, part of this goal is to prioritize those patients who most urgently need and can benefit the most from transplantation. Studying outcomes for each stratum based on a score utilizing $\mathrm{HCC}$ characteristics can help identify those patients and help to further calibrate HCC-based scores.

In conclusion, despite improvement in equity for the first six months under the delay, reverting to scheduled exception point progression after six months continues to advantage HCC patients. Thus an HCC-specific prioritization method, particularly for use after six months, is still needed. Utilizing the $M E L D_{E Q}$ after six months would improve equity of transplant probabilities between $\mathrm{HCC}$ and non-HCC patients, and its use through six months yields results similar to those of the six-month delay. Further, while overall projected dropout probabilities were nearly identical for the MELD $D_{E Q}$ and 6-month delay, projected differences between $\mathrm{HCC}$ and non-HCC patients were smaller under the MELD $D_{E Q}$. However, using the MELD ${ }_{E Q}$ is projected to increase dropout beyond non-HCC probabilities for some HCC risk strata. Therefore, further adjustment of the score would be needed before consideration for use in practice. 


\section{CHAPTER 3 \\ MELD $D_{E Q}$ AND deMELD COMPARISON}

For this study, two of the aforementioned equivalent MELD scores, the MELD ${ }_{E Q}{ }^{15}$ and the mixed new dropout equivalent MELD ${ }^{13}$ (referred to in this chapter as simply "deMELD”) were evaluated. Recall from Chapter 1 that these scores were derived by determining dropout probabilities or hazards based on established $\mathrm{HCC}$ characteristics as well as laboratory MELD, and then equating these HCC probabilities or hazards to those of non-HCC patients to find the corresponding MELD score. Both deMELD and MELD $D_{E Q}$ scores could be used for HCC patients comparably to the laboratory MELD for non-HCC patients since they are on the same scale. The deMELD was designed according to the probability of dropout from the waiting list while the $M E L D_{E Q}$ equated dropout hazards.

The primary aims of the current study were to compare the predictive accuracy for waitlist dropout of both scores and to evaluate possible effects of prioritization with these scores on waitlist dropout and transplant probabilities. These outcomes were studied in both original publications ${ }^{13,15}$, but the intent here was to validate the scores with newer data and to compare the scores with each other. Secondary goals were to study how changes in HCC-related covariates affected both alternative MELD scores, and compare post-transplant survival between patients in various ranges of the scores. 


\section{METHODS}

The same datasets for $\mathrm{HCC}$ and non-HCC patients as described in Chapter 2 were used for this study as well.

\section{Outcomes and Covariates}

The main outcomes studied included actual dropout and transplant probabilities for $\mathrm{HCC}$ patients under the scheduled progression in place during the timeframe of the study compared to those of non-HCC patients and those probabilities projected under the deMELD and MELD $\mathrm{EQ}_{\mathrm{EQ}}$. Also, post-transplant survival of patients stratified by ranges of the two alternative scores was evaluated. In addition, the effect of differences in the included covariates on categorization by the two alternative scoring models was examined. The covariates from these two models were alpha-fetoprotein (AFP) or the natural log of AFP, laboratory MELD, maximum tumor size, and number of tumors.

Additionally, the MELD $D_{E Q}$ calculation includes a constant which increases when waitlist time reaches six months.

Dropout, transplant and censored were defined in the same manner as the study described in Chapter 2 .

\section{Statistical Methods}

All data analysis and statistical calculations were performed using SAS (Version 9.4, Cary, NC) and R (Version 3.1.1). The MELD $\mathrm{EQ}_{\mathrm{EQ}}$ and deMELD were calculated for each observation of HCC patients in the dataset using the following equations:

$$
\begin{aligned}
\operatorname{MELD}_{\mathrm{EQ}}= & \max \left(\text { lab MELD, } 1.143^{*} \mathrm{MELD}+1.324^{*} \operatorname{In}(\mathrm{AFP})\right. \\
& \left.+1.438^{*} \text { Number of Tumors }+1.194^{*} \text { Max Tumor Size }+\mathrm{c}(\mathrm{t})\right)
\end{aligned}
$$


where $c(t)=-2 / 0.146$ for $\mathrm{t}<6$ months and $\mathrm{c}(\mathrm{t})=-1 / 0.146$ for $\mathrm{t} \geq 6$ months

$$
\begin{aligned}
\text { deMELD }= & \max \left(\text { lab MELD },-37.8+1.9^{\star}\right. \text { MELD } \\
& +5.9 \text { if Number of Tumors } \geq 2 \\
& +21.2 \text { if Max Tumor Size }>1 \mathrm{~cm}+5.9 \text { if AFP }>400)
\end{aligned}
$$

AFP values less than 1 were taken to be 1 for the purpose of calculating In(AFP). The observations were then categorized into ranges based on their $M_{E L D}$ and deMELD. These ranges included $<12,12-15,16-18,19-21,22-24$, 25-30, 31-35, and 36-40. These categories were then used as the transient states in nonparametric multistate models for dropout and transplant probabilities, using the R package msSurv. The multistate model is discussed in detail in the paper describing the msSurv package by Ferguson et al ${ }^{21}$. Figure 5 depicts a diagram of the multistate model used for this study. This is similar to the multistate model used in Chapter 2, but this model has eight transient states since eight risk strata were created as described above. The terminal states again are dropout and transplant.

Time zero was taken to be time first entering a given score range. This approach was used because clinically, projections for a patient given their current score or risk level are of interest in addition to their risk at listing. Also, very few patients were categorized in the highest risk groups at listing by either alternative score, as shown in Table 4. 


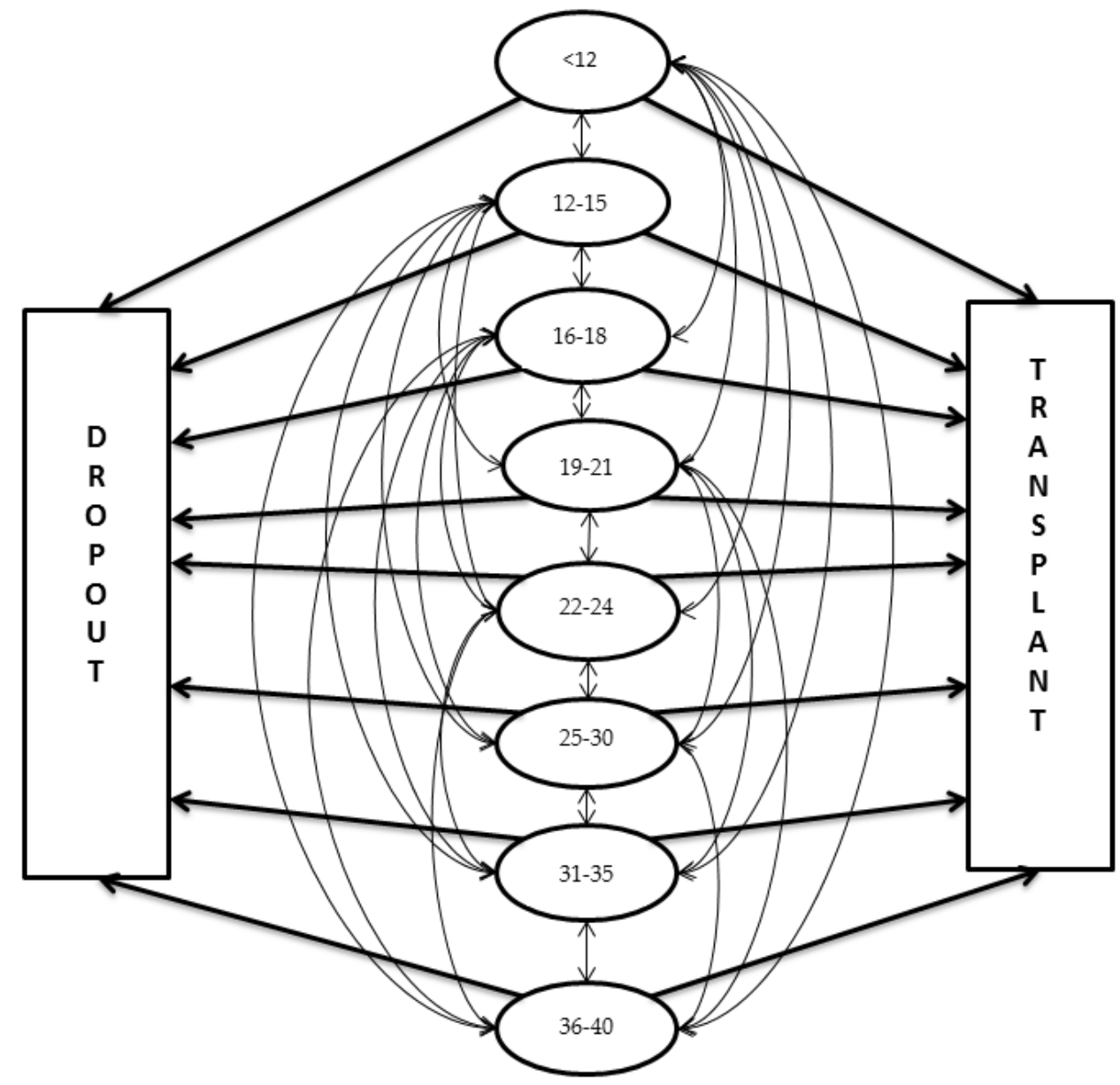

Figure 5: Schematic Diagram of Multistate Model. Transient states consisting of MELD or alternative MELD ranges are represented by ovals, while the terminal states of dropout and transplant are represented by rectangles. Transitions are possible between any of the transient states and another transient state (light lines), and from any transient state to the terminal states (bold lines). Transitions to the dropout / transplant states are of primary interest. 


\begin{tabular}{l|ll|ll}
\hline \multirow{2}{*}{$\begin{array}{l}\text { Score } \\
\text { Ranges }\end{array}$} & \multicolumn{2}{|c|}{ HCC MELD } & \multicolumn{2}{c}{ deMELD } \\
\hline $6-11$ & $4759(60.0)$ & $4081(51.5)$ & $4861(61.3)$ & $4580(57.8)$ \\
$12-15$ & $2075(26.2)$ & $2021(25.5)$ & $1803(22.7)$ & $1814(22.9)$ \\
$16-18$ & $692(8.7)$ & $933(11.8)$ & $676(8.5)$ & $766(9.7)$ \\
$19-21$ & $267(3.4)$ & $469(5.9)$ & $250(3.2)$ & $279(3.5)$ \\
$22-24$ & $103(1.3)$ & $226(2.9)$ & $160(2.0)$ & $222(2.8)$ \\
$25-30$ & $29(0.4)$ & $155(2.0)$ & $134(1.7)$ & $173(2.2)$ \\
$31-35$ & $1(0.01)$ & $32(0.4)$ & $31(0.4)$ & $56(0.7)$ \\
$36-40$ & $2(0.03)$ & $11(0.1)$ & $13(0.2)$ & $38(0.5)$ \\
\hline
\end{tabular}

Table 4. Frequency distributions of the MELD ${ }_{E Q}$ and deMELD scores for HCC patients at first listing and at last follow-up on the waitlist. Numbers in each cell are the count and percentage of patients out of 7928 total HCC patients.

To project dropout and transplant probabilities based on the proposed schemes, the same multistate model was constructed for non-HCC patients using analogous laboratory MELD score ranges, and the transplant hazard rates from the non-HCC model were substituted into the HCC models for groups in the same MELD $D_{E Q}$ and deMELD ranges. This is the same procedure that was used in the original paper describing the MELD $D_{E Q}{ }^{15}$. These were then used to project transplant probabilities using the Aalen-Johansen estimator ${ }^{21,22}$.

To compare equity of projected $\mathrm{HCC}$ dropout and transplant under the equivalent MELD models with that of non-HCC patients, matching of projected $\mathrm{HCC}$ transplant and dropout probabilities under each scheme to non-HCC probabilities was assessed graphically. Projected dropout was also assessed numerically for each stratum. The absolute value of the difference between nonHCC probabilities and projected HCC dropout probabilities was calculated. 
Similarly, the relative difference was calculated as a proportion of non-HCC probabilities. These absolute and relative differences were then averaged over all times through one year. To obtain an overall estimate of projected comparability to non-HCC probabilities for each score, an average of these absolute and relative differences was taken at each time, weighted by the proportion in each stratum of the number at risk at that time. These results were then averaged over all times through one year. Since the absolute value of these differences represents HCC dropout either exceeding or not reaching non-HCC dropout, a smaller value indicates better equality with non-HCC dropout probabilities.

Using the described score ranges, differences in the way the two alternative scores classified patients were explored by comparing the values of patient covariates for those observations where the alternative score categories agreed with those observations where they did not. The concordance index was also used to compare overall predictive accuracy for waitlist dropout ${ }^{27}$ between the two scores. Lastly, we compared post-transplant survival between the various risk groups as determined by both scores using Kaplan-Meier curves and Cox proportional hazard models.

\section{RESULTS}

A total of $7,928 \mathrm{HCC}$ and 34,868 non-HCC patients were studied as in Chapter 2. Table 4 shows the distributions of the two scores at initial listing and last follow-up. The overall C-indices for HCC waitlist dropout were 0.586 (95\% Cl: $0.562,0.61)$ for exception MELD scores, $0.653(95 \% \mathrm{Cl}: 0.624,0.682)$ for 
laboratory MELD, $0.678(95 \% \mathrm{Cl}: 0.649,0.707)$ for $M E L D_{E Q}$ and $0.664(95 \% \mathrm{Cl}$ : $0.635,0.693)$ for deMELD. In comparison, the C-index for non-HCC waitlist dropout was $0.832(95 \% \mathrm{Cl}: 0.822,0.842)$ for MELD.

To assess the agreement in actual dropout probabilities between nonHCC patients and HCC patients stratified by the MELD ${ }_{E Q}$, Figures $6 \mathbf{a}$ and $7 \mathbf{a}$ compare actual dropout probabilities for HCC patients stratified by MELD $D_{E Q}$ ranges to that of non-HCC patients in corresponding MELD ranges. For the lower risk categories (scores <22) shown in $\mathbf{6 a}$, the MELDEQ matches non-HCC dropout fairly well until about 6 months from entering a state and then begins not to reach non-HCC dropout probabilities, especially for the 16-18 and 19-21 score ranges. As shown on Figure 7a, the actual dropout probability curve for the $M E L D_{E Q} 22-24$ group matches the corresponding non-HCC curve well at most times, but matching is poor for groups with MELD $\mathrm{EQ}_{\mathrm{Q}}$ scores above 24 . There is some matching for the $36-40$ group but data are sparse in this range.

A similar assessment of agreement in actual dropout probabilities was performed for the deMELD (Figures 8a and 9a). The deMELD showed good matching through about eight months from entering a state for scores $<22$ (Figure 8a). Matching of actual dropout probabilities to corresponding non-HCC ranges is somewhat better under the deMELD than the MELD $D_{E Q}$ for scores less than 22. Matching is poor under deMELD for groups with scores greater than 21 (Figure 8a). Again, data are sparse for the higher risk groups, especially as stratified by the MELD $D_{E Q}$. This can be seen in the numbers at risk shown in Figures $7 a$ and $9 a$. 
Projected dropout probabilities are increased beyond target non-HCC levels for groups in the lower risk ranges $\left(M E L D_{E Q}\right.$ and deMELD $\left.<16\right)$ under both alternative scores (see Figures $\mathbf{6 b}$ and $\mathbf{8 b}$ ), though to a lesser degree for deMELD 12-15 (Figure 8b). However, dropout would decrease for those with alternative MELD scores $\geq 22$ (Figures $7 \mathbf{b}$ and $9 \mathbf{b}$ ), to a lesser degree for $M E L D_{E Q} 22-24$. Actual dropout is already lower than non-HCC for most of these strata (Figures 7a and 9a). Actual and projected dropout probabilities appear especially low for deMELD $\geq 22$ (Figures $9 a$ and $9 b$ ).

Table 5 displays absolute and relative differences by stratum and overall average difference (weighted by strata) between non-HCC dropout and projected HCC dropout under each equivalent MELD score if implemented. Matching to non-HCC dropout probabilities is similar between the deMELD and the MELD $\mathrm{EQ}_{\mathrm{E}}$ for equivalent scores $<12,16-18$, and 31-35. Matching is better for deMELD scores of 12-15 (absolute difference of 0.00647 vs. 0.01152 for $M E L D_{E Q}$ ) and 19$21\left(0.00564\right.$ vs 0.00745 for MELD $\left.D_{E Q}\right)$. Matching is better for MELD $D_{E Q}$ scores of $22-24$ (0.01178 vs. 0.04148 for deMELD), $25-30$ (0.04693 vs. 0.06388 for deMELD), and 35-40 (0.06820 vs. 0.15135 for deMELD). The weighted overall difference is very similar between the two scores.

Projected transplant for both scores (Figures 6-9, panel d) shows better stratification based on risk level than actual transplant probabilities (Figures 6-9, panel c), especially for the lower risk groups, although lower than target non$\mathrm{HCC}$ probabilities for most groups. The MELD $\mathrm{EQ}_{\mathrm{E}}$ matches non-HCC transplant probabilities slightly better than the deMELD for equivalent scores $12-15$ and similarly to the deMELD for scores $<12$ and $16-18$. The deMELD score matches 
non-HCC transplant probabilities better than the MELD $D_{E Q}$ for scores $>18$ and particularly scores $\geq 31$. However, neither score matches especially closely for the range of 19-30 (Figures 6-9, panel d).

\begin{tabular}{l|l|r|l|r}
\hline $\begin{array}{l}\text { Equivalent } \\
\text { MELD } \\
\text { Score }\end{array}$ & \multicolumn{2}{|c|}{ MELD $_{\mathrm{EQ}}$} & \multicolumn{2}{c}{ deMELD } \\
Range & \multicolumn{1}{|c|}{ Difference from non-HCC } & \multicolumn{1}{c}{$\begin{array}{l}\text { Difference from non-HCC } \\
\text { Relative }\end{array}$} \\
\hline$<12$ & Absolute & Relative & Absolute & $41.4 \%$ \\
$12-15$ & 0.01002 & $39.7 \%$ & 0.01049 & $18.4 \%$ \\
$16-18$ & 0.01152 & $29.8 \%$ & 0.00647 & $6.9 \%$ \\
$19-21$ & 0.00236 & $6.9 \%$ & 0.00239 & $9.4 \%$ \\
$22-24$ & 0.00745 & $9.5 \%$ & 0.00564 & $38.9 \%$ \\
$25-30$ & 0.01178 & $12.0 \%$ & 0.04148 & $50.4 \%$ \\
$31-35$ & 0.04693 & $37.4 \%$ & 0.06388 & $60.6 \%$ \\
$35-40$ & 0.09742 & $63.4 \%$ & 0.09372 & $75.8 \%$ \\
Overall & 0.06820 & $35.9 \%$ & 0.15135 & $32.6 \%$ \\
\hline
\end{tabular}

Table 5. Average absolute and relative differences between non-HCC and projected HCC dropout probabilities under MELD $\mathrm{EQ}_{\mathrm{Q}}$ or deMELD by range of each equivalent MELD score. The absolute differences are the absolute values of the difference between the projected HCC dropout curves and non-HCC actual dropout curves depicted in Figures $\mathbf{6 b}, \mathbf{7 b}, \mathbf{8 b}$ and $\mathbf{9 b}$. The relative differences are those absolute differences as a proportion of actual non-HCC dropout probabilities. Smaller values indicate closer matching between the equivalent MELD range and the target non-HCC MELD range. The overall differences weighted by number at risk in each stratum and averaged over all times through 365 days are shown in the bottom row. The number at risk is shown for $0,3,6$ and 9 months on Figures $\mathbf{6 a}, \mathbf{7 a}, \mathbf{8} \mathbf{a}$ and $\mathbf{9 a}$, though for this calculation the number at risk at each time from 1 to 365 days was used. 

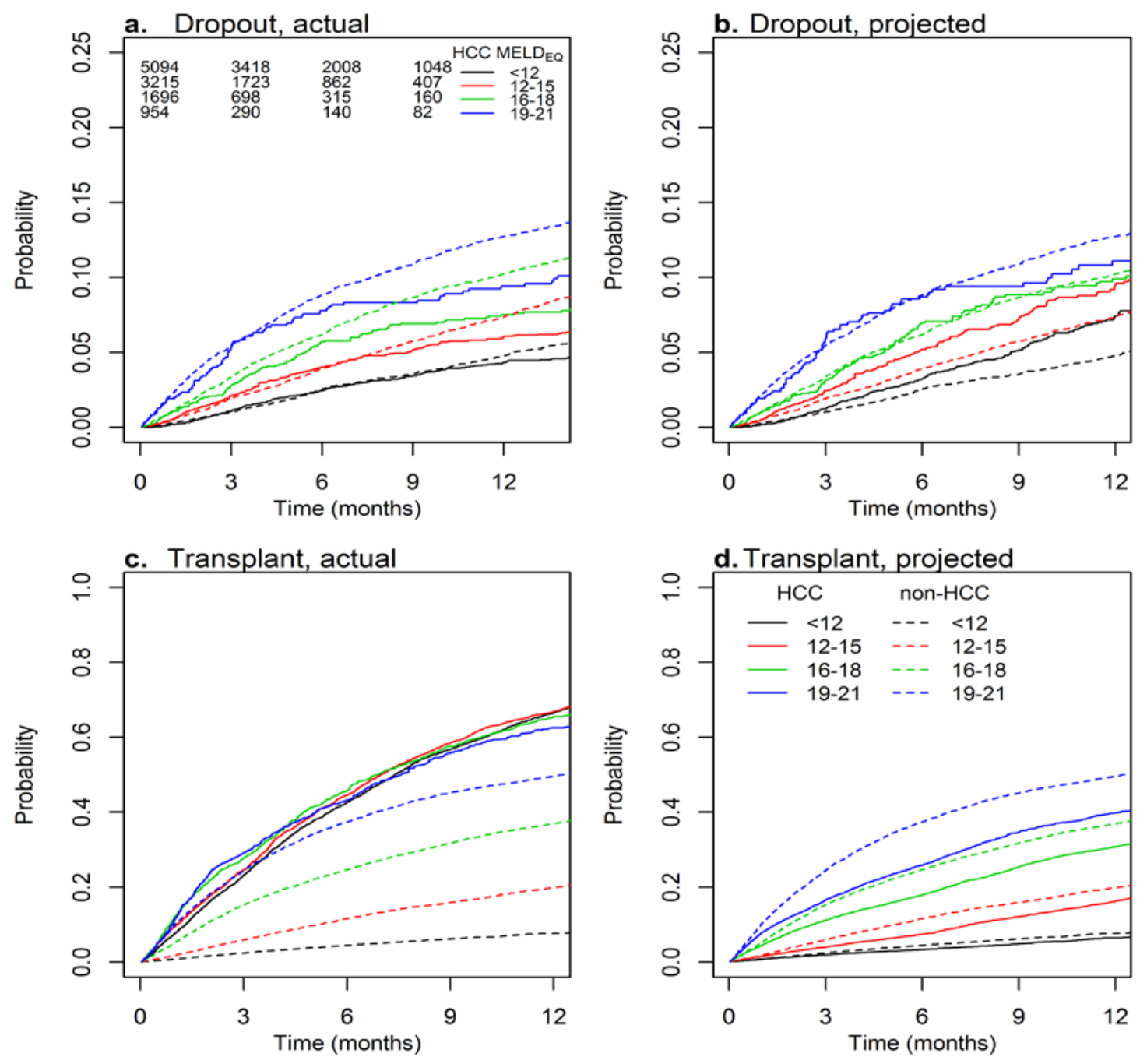

Figure 6: Actual and projected time to dropout/transplant for lower-risk $\left(M_{E L D}<22\right) H C C$ patients stratified by MELD $D_{E Q}$ score. Time is from entry into the corresponding MELD ${ }_{E Q}$ range for $\mathrm{HCC}$ patients or the MELD range for non-HCC patients. Solid lines indicate probablity of transplant/dropout for HCC patients, whereas dashed lines indicate corresponding probability curves (actual only) for non-HCC patients. The number at risk for $\mathrm{HCC}$ patients at $0,3,6$, and 9 months is given in panel a. The predictions pertain only to HCC patients; for non-HCC patients only actual probability curves are given. 

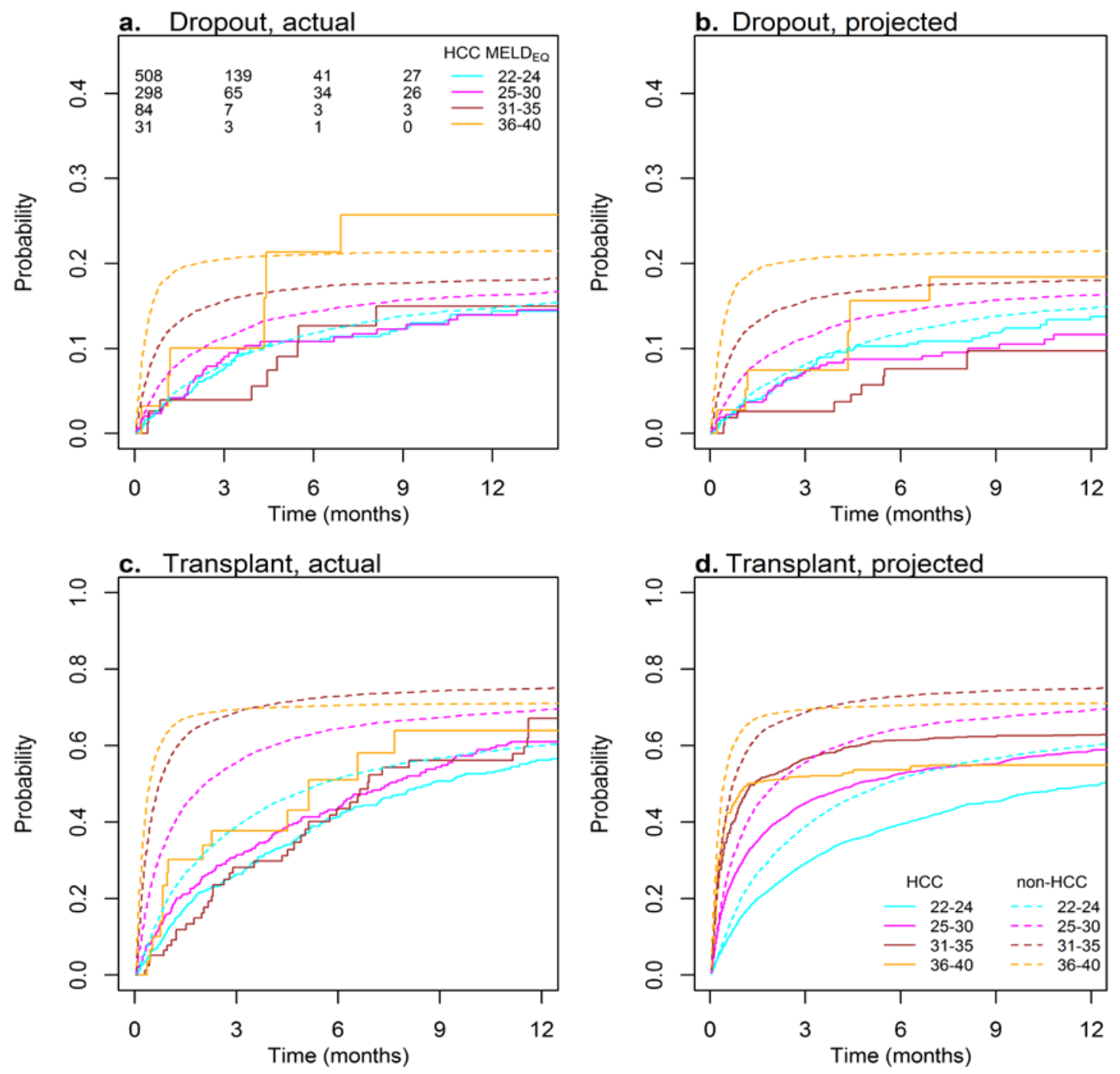

Figure 7: Actual and projected time to dropout/transplant for higher-risk $\left(\right.$ MELD $\left._{E Q} \geq 22\right)$ HCC patients stratified by MELD ${ }_{E Q}$ score. Time is from entry into the corresponding MELD ${ }_{E Q}$ range for $\mathrm{HCC}$ patients or the MELD range for non-HCC patients. Solid lines indicate probablity of transplant/dropout for HCC patients, whereas dashed lines indicate corresponding probability curves (actual only) for non-HCC patients. The number at risk for $\mathrm{HCC}$ patients at $0,3,6$, and 9 months is given in panel a. The predictions pertain only to HCC patients; for non-HCC patients only actual probability curves are given. 

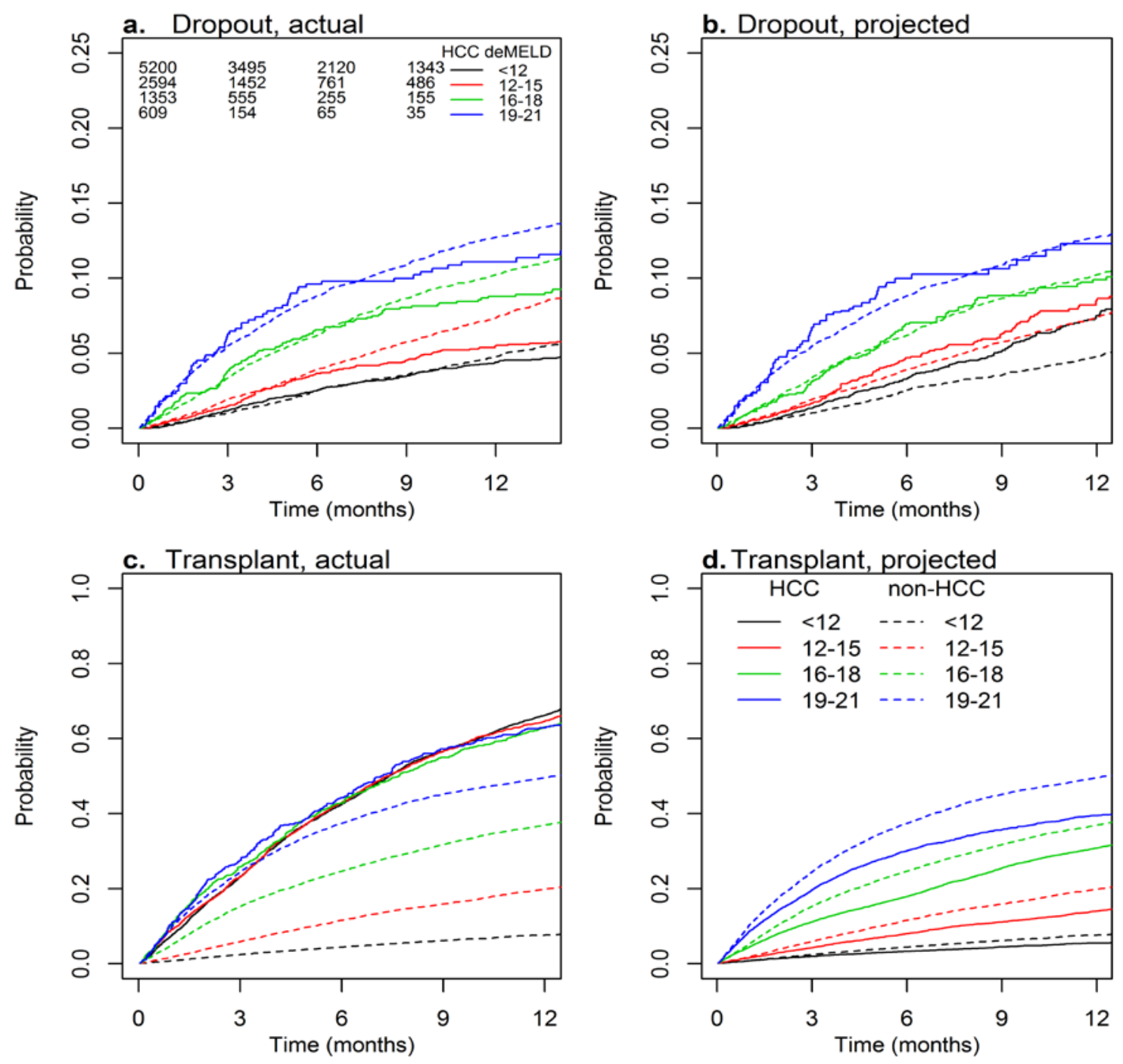

Figure 8: Actual and projected time to dropout/transplant for lower-risk (deMELD<22) HCC patients stratified by deMELD score. Time is from entry into the corresponding deMELD range for HCC patients or the MELD range for non-HCC patients. Solid lines indicate probablity of transplant/dropout for HCC patients, whereas dashed lines indicate corresponding probability curves (actual only) for non-HCC patients. The number at risk for $\mathrm{HCC}$ patients at $0,3,6$, and 9 months is given in panel a. The predictions pertain only to HCC patients; for non-HCC patients only actual probability curves are given. 

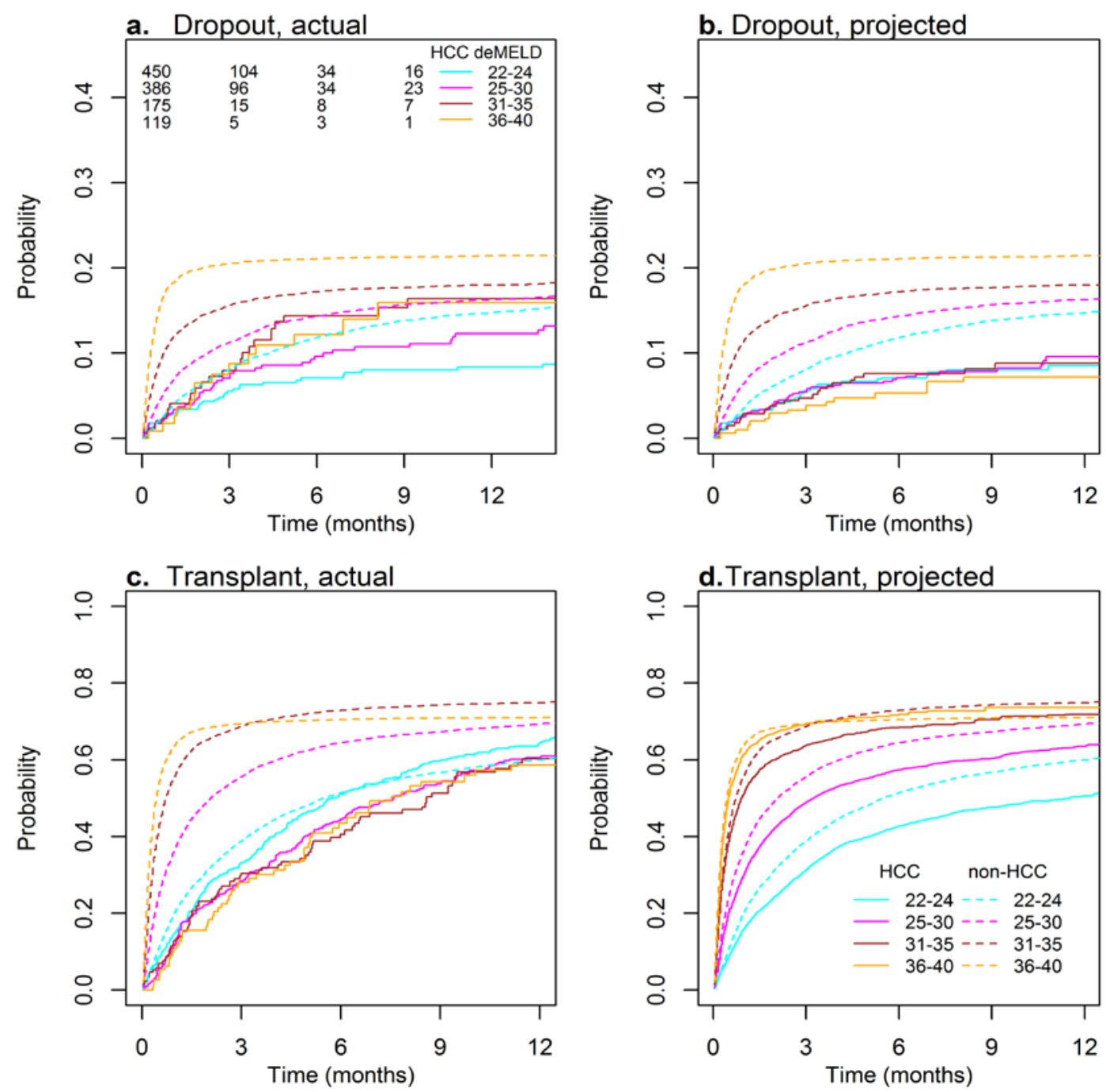

Figure 9: Actual and projected time to dropout/transplant for higher-risk (deMELD $\geq 22$ ) HCC patients stratified by deMELD score. Time is from entry into the corresponding deMELD range for HCC patients or the MELD range for non-HCC patients. Solid lines indicate probablity of transplant/dropout for HCC patients, whereas dashed lines indicate corresponding probability curves (actual only) for non-HCC patients. The number at risk for $\mathrm{HCC}$ patients at $0,3,6$, and 9 months is given in panel a. The predictions pertain only to HCC patients; for non-HCC patients only actual probability curves are given. 


\section{Effect of Covariates on MELDEQ and deMELD}

Table 6 shows a cross-tabulation of how each observation on the waiting list was categorized by each alternative score. Risk groups with MELD $D_{E Q}$ and deMELD scores $<19$ had the most agreement between the two scores: the majority of observations placed in these categories by one score were also placed there by the other score. Table 7 shows summaries of the contributing covariates for observations that were placed in the same category by both scores and Table 8 shows those that were categorized differently by the two alternative scores. This comparison was made when less than half the observations assigned to a category by one alternative score were also assigned there by the other score. For example, 411 observations had deMELD scores in the $36-40$ range, and $93(22.6 \%)$ of these also had MELD $D_{\mathrm{EQ}}$ scores of 36-40 (see Table 6). Another $171(41.6 \%)$ of them had MELD $D_{E Q}$ scores of $25-30$, so the covariates of these 93 observations with score agreement were compared with those of the 171 observations with score disagreement.

As shown in Tables 7 and $\mathbf{8}$, in groups where the deMELD category exceeded the MELDEQ category, less than half of observations had waitlist times of six months or more, with the exception of the highest risk group $\left(M E L D_{E Q}=\right.$ 31-35 and deMELD = 36-40, $n=147$, Table 8). In contrast, for the groups in which the MELD $D_{E Q}$ was at least as great as the deMELD score (outside of scores in the 19-21 range), $71-94.4 \%$ of waitlist times were greater than 6 months. The MELD $D_{E Q}$ assigns 6.85 more points when waitlist time is at least six months, and the deMELD does not include a term for time on the waitlist. Also, in the groups for where the deMELD category exceeded the MELD $D_{E Q}$ category, all 
observations had a maximum tumor size greater than $1 \mathrm{~cm}$, which was not true for categories with $M E L D_{E Q} \geq$ deMELD. A tumor size $>1 \mathrm{~cm}$ adds 21.2 points to the deMELD score, compared to 1.194 points for each $\mathrm{cm}$ increase for the MELD $D_{E Q}$

No obvious differences were noted in this comparison for number of tumors or AFP, so the distribution of them in the dataset as a whole was examined. For number of tumors, the deMELD assigns more points unless there is only 1 tumor or greater than 4 tumors, since the deMELD assigns 5.9 points for $\geq 2$ tumors and the MELD $E Q$ assigns 1.438 points per tumor. However, about $80 \%$ of observations in the dataset had only 1 tumor (note that each patient can have multiple observations). Thus, for the majority of observations, the MELD $\mathrm{EQ}_{\mathrm{Q}}$ was assigning 1.438 points for number of tumors while the deMELD was assigning 0 points, which is not a large difference when considering the other factors.

For AFP >1, the MELDEQ will assign more points than the deMELD, as the deMELD gains 5.9 points if AFP $>400$, and 0 points otherwise. If AFP $>400$ this term will be at least 7.93 for the MELD $D_{\mathrm{EQ}}:(\ln (401)=5.99$ so the AFP term for $M E L D_{E Q}$ will be $\left.5.99^{*} 1.324=7.93\right)$. However, if $\ln (A F P)=0$, neither score will gain points for AFP. Less than $25 \%$ of the observations in each group compared had AFP levels $>400$, and less than $15 \%$ had $\ln (A F P)=0$. Thus, the $M E L D_{E Q}$ assigned points (approximately 1 to 8 ) for AFP to most observations while the deMELD assigned 0 for most observations. In the entire dataset only 1698 $(3.2 \%)$ of observations had AFP $>400$ and only $845(1.6 \%)$ had $\ln ($ AFP $)=0$. The median AFP in the dataset was 9 , and the $75^{\text {th }}$ percentile was 29 , so half the 
observations would receive less than 2.9 points, and the majority would be assigned less than 4.5 points by the MELD $\mathrm{EQ}_{\mathrm{B}}$ for AFP.

For the laboratory MELD, it was noted that in the groups studied where the deMELD category exceeded the MELD $D_{E Q}$ category, none of the deMELD scores matched the laboratory MELD. The converse was also true for the category in which MELD $D_{E Q}$ exceeded deMELD, but only one such category was examined (Table 8). This is not surprising since each score takes the maximum of the calculated score or the laboratory MELD (so a higher equivalent score might already exceed the laboratory MELD). The deMELD assigns 1.9 points and the MELD $D_{E Q}$ assigns 1.143 points for every 1 point increase in laboratory MELD.

Of the groups compared for score agreement, waitlist time and maximum tumor size seemed to have the most effect, which is expected since these will generally account for the biggest differential in contributions to each score. 


\begin{tabular}{|c|c|c|c|c|c|c|c|c|c|}
\hline & deMEL & Categ & & & & & & & \\
\hline $\begin{array}{l}\text { MELD }_{\mathrm{EQ}} \\
\text { Category }\end{array}$ & $<12$ & $12-15$ & $16-18$ & $19-21$ & 22-24 & $25-30$ & $31-35$ & $36-40$ & $\begin{array}{r}\text { Row } \\
\text { Totals } \\
\end{array}$ \\
\hline$<12$ & $\begin{array}{l}27189 \\
99.9 \% \\
89.9 \%\end{array}$ & 24 & 2 & 0 & 0 & 0 & 0 & 0 & $\begin{array}{l}27215 \\
50.7 \% \\
\end{array}$ \\
\hline $12-15$ & 2552 & $\begin{array}{r}10640 \\
76 \% \\
82.7 \% \\
\end{array}$ & 789 & 13 & 1 & 0 & 0 & 0 & $\begin{array}{r}13995 \\
26.1 \% \\
\end{array}$ \\
\hline $16-18$ & 409 & 1608 & $\begin{array}{r}3269 \\
54.3 \% \\
64 \% \\
\end{array}$ & 342 & $\begin{array}{r}390 \\
6.5 \% \\
31.8 \%\end{array}$ & 2 & 0 & 0 & $\begin{array}{r}6020 \\
11.2 \%\end{array}$ \\
\hline $19-21$ & 57 & 543 & $\begin{array}{r}747 \\
23.6 \% \\
14.6 \% \\
\end{array}$ & $\begin{array}{r}1168 \\
36.9 \% \\
60.8 \% \\
\end{array}$ & 337 & 310 & 0 & 0 & $\begin{array}{l}3162 \\
5.9 \% \\
\end{array}$ \\
\hline $22-24$ & 12 & 55 & 261 & 297 & $\begin{array}{r}383 \\
22.3 \% \\
31.2 \% \\
\end{array}$ & $\begin{array}{r}530 \\
30.9 \% \\
39.1 \% \\
\end{array}$ & 176 & 0 & $\begin{array}{l}1714 \\
3.2 \% \\
\end{array}$ \\
\hline $25-30$ & 12 & 0 & 42 & 102 & 116 & $\begin{array}{r}480 \\
39.7 \% \\
35.4 \% \\
\end{array}$ & $\begin{array}{r}285 \\
23.6 \% \\
52.1 \% \\
\end{array}$ & $\begin{array}{r}171 \\
14.2 \% \\
41.6 \% \\
\end{array}$ & $\begin{array}{l}1208 \\
2.3 \% \\
\end{array}$ \\
\hline $31-35$ & 0 & 0 & 0 & 0 & 1 & 31 & $\begin{array}{r}80 \\
30.9 \% \\
14.6 \% \\
\end{array}$ & $\begin{array}{r}147 \\
56.8 \% \\
35.8 \% \\
\end{array}$ & $\begin{array}{r}259 \\
0.5 \%\end{array}$ \\
\hline $36-40$ & 0 & 0 & 0 & 0 & 0 & 2 & 6 & $\begin{array}{r}93 \\
92.1 \% \\
22.6 \% \\
\end{array}$ & $\begin{array}{r}101 \\
0.2 \% \\
\end{array}$ \\
\hline $\begin{array}{l}\text { Column } \\
\text { Totals }\end{array}$ & $\begin{array}{l}30231 \\
56.3 \% \\
\end{array}$ & $\begin{array}{r}12870 \\
24 \% \\
\end{array}$ & $\begin{array}{l}5110 \\
9.5 \% \\
\end{array}$ & $\begin{array}{l}1922 \\
3.6 \% \\
\end{array}$ & $\begin{array}{l}1228 \\
2.3 \% \\
\end{array}$ & $\begin{array}{l}1355 \\
2.5 \% \\
\end{array}$ & $\begin{array}{r}547 \\
1 \% \\
\end{array}$ & $\begin{array}{r}411 \\
0.8 \% \\
\end{array}$ & 53674 \\
\hline
\end{tabular}

Table 6: Cross tabulation of equivalent MELD score assignments by

MELD ${ }_{E Q}$ and deMELD. The numbers in each cell are the number of HCC

observations categorized by the deMELD score in the column heading and the

$M E L D_{E Q}$ score in the row heading followed by the row percent and column

percent. The row and column totals shown include the percent of total

observations. Each individual patient can have multiple observations

representing different follow up times on the waiting list. The cells where scoring

systems agree are green, and those selected for comparison where scores do

not agree are red. 


\begin{tabular}{|c|c|c|c|}
\hline $\begin{array}{ll} & \text { Comparison } \\
\text { Covariate } & \end{array}$ & $\begin{array}{l}\text { MELD }{ }_{E Q} \text { and deMELD=36-40 } \\
n=93 \text { obs. }\end{array}$ & $\begin{array}{l}\text { MELD }{ }_{E Q} \text { and deMELD=31-35 } \\
n=80 \text { obs. }\end{array}$ & $\begin{array}{l}\text { MELD }{ }_{E Q} \text { and deMELD }=25-30 \\
n=480 \text { obs. }\end{array}$ \\
\hline $\begin{array}{l}\text { Waitlist time } \\
+6.85 \text { to } M E L D_{E Q} \text { if } \geq 6 \mathrm{mo}\end{array}$ & $85(91.4 \%) \geq 6 \mathrm{mo}$ & $73(91.3 \%) \geq 6 \mathrm{mo}$ & $408(85 \%) \geq 6 \mathrm{mo}$ \\
\hline $\begin{array}{l}\text { Max tumor size } \\
+21.2 \text { to deMELD if }>1 \mathrm{~cm} \\
+1.194^{*} \text { size to MELD }{ }_{\mathrm{EQ}}\end{array}$ & $\begin{array}{l}85(91.4 \%)>1 \mathrm{~cm} \\
8(8.6 \%) \text { were } 0 \\
\text { Median size }=2.4 \mathrm{~cm}\end{array}$ & $\begin{array}{l}60(75 \%)>1 \mathrm{~cm} \\
16(20 \%) \text { were } 0 \\
\text { Median size }=2.3 \mathrm{~cm}\end{array}$ & $\begin{array}{l}383(79.8 \%)>1 \mathrm{~cm} \\
88(18.3 \%) \text { were } 0 \\
\text { Median size }=2 \mathrm{~cm}\end{array}$ \\
\hline $\begin{array}{l}\text { Number of tumors } \\
+5.9 \text { to deMELD if } \geq 2 \\
+1.438^{*} \text { no. to } M E L D_{\mathrm{EQ}}\end{array}$ & $\begin{array}{l}45(48.4 \%) \geq 2 \\
\text { none were } 0 \\
\text { Median no. }=1\end{array}$ & $\begin{array}{l}12(15 \%) \geq 2 \\
\text { none were } 0 \\
\text { Median no. }=1\end{array}$ & $\begin{array}{l}134(27.9 \%) \geq 2 \\
\text { none were } 0 \\
\text { Median no. }=1\end{array}$ \\
\hline $\begin{array}{l}\text { AFP or } \ln (\text { AFP }) \\
+5.9 \text { to deMELD if }>400 \\
+1.324^{*} \ln (\text { AFP }) \text { to } M E L D_{E Q}\end{array}$ & $\begin{array}{l}21(22.6 \%) w / A F P>400 \\
1(1.1 \%) w / \ln (A F P)=0 \\
\text { Median } n(A F P)=2.1\end{array}$ & $\begin{array}{l}14(17.5 \%) w / A F P>400 \\
\text { none } w / \ln (A F P)=0 \\
\text { Median } \ln (A F P)=3\end{array}$ & $\begin{array}{l}54(11.3 \%) w / A F P>4003 \\
(<1 \%) w / \ln (A F P)=0 \\
\text { Median } \ln (\text { AFP })=2.6\end{array}$ \\
\hline $\begin{array}{l}\text { Lab MELD } \\
+1.9^{*} \text { MELD to deMELD } \\
+1.143^{*} \text { MELD to MELD }{ }_{\mathrm{EQ}}\end{array}$ & $\begin{array}{l}\text { Range } 22-40 \\
12(12.9 \%) w / M E L D=d e M E L D \\
13(14 \%) w / M E L D=M E L D_{E Q}\end{array}$ & $\begin{array}{l}\text { Range } 20-35 \\
20(25 \%) w / M E L D=\text { deMELD } \\
12(15 \%) w / M E L D=M E L D_{E Q}\end{array}$ & $\begin{array}{l}\text { Range } 16-30 \\
97(20.2 \%) w / M E L D=d e M E L D \\
73(15.2 \%) w / M E L D=M E L D_{E Q}\end{array}$ \\
\hline
\end{tabular}

\begin{tabular}{|c|c|c|}
\hline $\begin{array}{ll}\text { Covariate } & \text { Comparison } \\
\end{array}$ & $\begin{array}{l}\text { MELD }{ }_{E Q} \text { and deMELD=22-24 } \\
n=383 \text { obs. }\end{array}$ & $\begin{array}{l}\text { MELD }{ }_{E Q} \text { and deMELD=19-21 } \\
n=1168 \text { obs. }\end{array}$ \\
\hline $\begin{array}{l}\text { Waitlist time } \\
+6.85 \text { to } M E L D_{E Q} \text { if } \geq 6 \text { mo }\end{array}$ & $272(71 \%) \geq 6 \mathrm{mo}$ & $391(33.5 \%) \geq 6 \mathrm{mo}$ \\
\hline $\begin{array}{l}\text { Max tumor size } \\
+21.2 \text { to deMELD if }>1 \mathrm{~cm} \\
+1.194^{*} \text { size to MELD }{ }_{\mathrm{EQ}}\end{array}$ & $\begin{array}{l}176(46 \%)>1 \mathrm{~cm} \\
186(48.6 \%) \text { were } 0 \\
\text { Median size }=0.8 \mathrm{~cm}\end{array}$ & $\begin{array}{l}790(67.6 \%)>1 \mathrm{~cm} \\
342(29.4 \%)) \text { were } 0 \\
\text { Median size }=2 \mathrm{~cm}\end{array}$ \\
\hline $\begin{array}{l}\text { Number of tumors } \\
+5.9 \text { to deMELD if } \geq 2 \\
+1.438^{*} \text { no. to } M E L D_{E Q}\end{array}$ & $\begin{array}{l}96(25.1 \%) \geq 2 \\
\text { none were } 0 \\
\text { Median no. }=1\end{array}$ & $\begin{array}{l}109(9.3 \%) \geq 2 \\
20(1.7 \%) \text { were } 0 \\
\text { Median no. }=1\end{array}$ \\
\hline $\begin{array}{l}\text { AFP or } \ln (\text { AFP }) \\
+5.9 \text { to deMELD if }>400 \\
+1.324^{*} \ln (\text { AFP }) \text { to } M E L D_{E Q}\end{array}$ & $\begin{array}{l}11(2.9 \%) w / A F P>400 \\
14(3.7 \%) w / \ln (A F P)=0 \\
\text { Median } \ln (A F P)=1.8\end{array}$ & $\begin{array}{l}35(3 \%) w / A F P>400 \\
35(3 \%) w / \ln (A F P)=0 \\
\text { Median } \ln (A F P)=1.6\end{array}$ \\
\hline $\begin{array}{l}\text { Lab MELD } \\
+1.9^{*} \text { MELD to deMELD } \\
+1.143^{*} \text { MELD to MELD }{ }_{\mathrm{EQ}}\end{array}$ & $\begin{array}{l}\text { Range } 14-24 \\
207(54 \%) w / M E L D=d e M E L D \\
178(46.5 \%) w / M E L D=M E L D_{E Q}\end{array}$ & $\begin{array}{l}\text { Range } 13-21 \\
378(32.4 \%) \mathrm{w} / \mathrm{MELD}=\text { deMELD } \\
890(76.2 \%) \mathrm{w} / \mathrm{MELD}=\mathrm{MELD}{ }_{\mathrm{EQ}}\end{array}$ \\
\hline
\end{tabular}

Table 7. Effect of covariates with score agreement. 


\begin{tabular}{|c|c|c|c|}
\hline Covariate $\quad$ Comparison & $\begin{array}{l}\text { MELD }{ }_{E Q}=31-35, \text { deMELD=36-40 } \\
n=147 \text { obs. }\end{array}$ & $\begin{array}{l}\text { MELD } D_{E Q}=25-30, \text { deMELD=36-40 } \\
n=171 \text { obs. }\end{array}$ & $\begin{array}{l}M E L D_{E Q}=25-30, \text { deMELD=31-35 } \\
n=285 \text { obs. }\end{array}$ \\
\hline $\begin{array}{l}\text { Waitlist time } \\
+6.85 \text { to } M E L D_{E Q} \text { if } \geq 6 \text { mo }\end{array}$ & $109(74 \%) \geq 6 \mathrm{mo}$ & $9(5.3 \%) \geq 6 \mathrm{mo}$ & $130(45.6 \%) \geq 6 \mathrm{mo}$ \\
\hline $\begin{array}{l}\text { Max tumor size } \\
+21.2 \text { to deMELD if }>1 \mathrm{~cm} \\
+1.194^{*} \text { size to } M E L D_{\mathrm{EQ}}\end{array}$ & $\begin{array}{l}147(100 \%)>1 \mathrm{~cm} \\
\text { None were } 0 \\
\text { Median size }=2 \mathrm{~cm}\end{array}$ & $\begin{array}{l}171(100 \%)>1 \mathrm{~cm} \\
\text { None were } 0 \\
\text { Median size }=2.1 \mathrm{~cm}\end{array}$ & $\begin{array}{l}285(100 \%)>1 \mathrm{~cm} \\
\text { None were } 0 \\
\text { Median size }=2.2 \mathrm{~cm}\end{array}$ \\
\hline $\begin{array}{l}\text { Number of tumors } \\
+5.9 \text { to deMELD if } \geq 2 \\
+1.438^{*} \text { no. to } M E L D_{\mathrm{EQ}}\end{array}$ & $\begin{array}{l}61(41.5 \%) \geq 2 \\
\text { None were } 0 \\
\text { Median no. }=1\end{array}$ & $\begin{array}{l}109(63.7 \%) \geq 2 \\
2(1.2 \%) \text { were } 0 \\
\text { Median no. }=2\end{array}$ & $\begin{array}{l}102(35.8 \%) \geq 2 \\
\text { None were } 0 \\
\text { Median no. }=1\end{array}$ \\
\hline $\begin{array}{l}\text { AFP or } \ln (\text { AFP }) \\
+5.9 \text { to deMELD if }>400 \\
+1.324^{*} \ln (\text { AFP }) \text { to } M E L D_{E Q}\end{array}$ & $\begin{array}{l}9(6.1 \%) w / A F P>400 \\
15(10.2 \%) w / \ln (A F P)=0 \\
\text { Median } \ln (A F P)=1.8\end{array}$ & $\begin{array}{l}3(1.8 \%) w / A F P>400 \\
6(3.5 \%) w / \ln (A F P)=0 \\
\text { Median } \ln (A F P)=2.2\end{array}$ & $\begin{array}{l}22(7.7 \%) w / A F P>400 \\
20(7 \%) w / \ln (A F P)=0 \\
\text { Median } \ln (\text { AFP })=2.3\end{array}$ \\
\hline $\begin{array}{l}\text { Lab MELD } \\
+1.9^{*} \text { MELD to deMELD } \\
+1.143^{*} \text { MELD to MELD }{ }_{\mathrm{EQ}}\end{array}$ & $\begin{array}{l}\text { Range } 22-35 \\
0 \text { w/MELD = deMELD } \\
29(19.7 \%) \text { w/MELD=MELD } \\
\text { EQ }\end{array}$ & $\begin{array}{l}\text { Range } 22-30 \\
0 \text { w/MELD = deMELD } \\
153(89.5 \%) \text { w/MELD=MELD }{ }_{E Q}\end{array}$ & $\begin{array}{l}\text { Range } 20-27 \\
0 \text { w/MELD = deMELD } \\
109(38.2 \%) \text { w/MELD=MELD } \\
\text { EQ }\end{array}$ \\
\hline
\end{tabular}

\begin{tabular}{|c|c|c|c|}
\hline Covariate Comparison & $\begin{array}{l}M E L D_{E Q}=22-24, \text { deMELD=25-30 } \\
n=530 \text { obs. }\end{array}$ & $\begin{array}{l}\text { MELD } D_{E Q}=16-18, \text { deMELD=22-24 } \\
n=390 \text { obs. }\end{array}$ & $\begin{array}{l}\text { MELD }_{\mathrm{EQ}}=19-21, \text { deMELD }=16-18 \\
\mathrm{n}=747 \text { obs. }\end{array}$ \\
\hline $\begin{array}{l}\text { Waitlist time } \\
+6.85 \text { to } M E L D_{E Q} \text { if } \geq 6 \text { mo }\end{array}$ & $120(22.6 \%) \geq 6 \mathrm{mo}$ & $14(3.6 \%) \geq 6 \mathrm{mo}$ & $705(94.4 \%) \geq 6 \mathrm{mo}$ \\
\hline $\begin{array}{l}\text { Max tumor size } \\
+21.2 \text { to deMELD if }>1 \mathrm{~cm} \\
+1.194^{*} \text { size to MELD } \\
\end{array}$ & $\begin{array}{l}530(100 \%)>1 \mathrm{~cm} \\
\text { None were } 0 \\
\text { Median size }=2.4 \mathrm{~cm}\end{array}$ & $\begin{array}{l}390(100 \%)>1 \mathrm{~cm} \\
\text { None were } 0 \\
\text { Median size }=2 \mathrm{~cm}\end{array}$ & $\begin{array}{l}682(91.3 \%)>1 \mathrm{~cm} \\
61(8.2 \%) \text { were } 0 \\
\text { Median size }=2.2 \mathrm{~cm}\end{array}$ \\
\hline $\begin{array}{l}\text { Number of tumors } \\
+5.9 \text { to deMELD if } \geq 2 \\
+1.438^{*} \text { no. to } M E L D_{E Q}\end{array}$ & $\begin{array}{l}91(17.2 \%) \geq 2 \\
1(0.2 \%) \text { was } 0 \\
\text { Median no. }=1\end{array}$ & $\begin{array}{l}382(97.9 \%) \geq 2 \\
\text { None were } 0 \\
\text { Median no. }=2\end{array}$ & $\begin{array}{l}154(20.6 \%) \geq 2 \\
8(1.1 \%) \text { were } 0 \\
\text { Median no. }=1\end{array}$ \\
\hline $\begin{array}{l}\text { AFP or } \ln (\text { AFP }) \\
+5.9 \text { to deMELD if }>400 \\
+1.324^{*} \ln (\text { AFP }) \text { to } M E L D D_{\mathrm{EQ}}\end{array}$ & $\begin{array}{l}22(4.2 \%) w / A F P>400 \\
33(6.2 \%) w / \ln (A F P)=0 \\
\text { Median } \ln (A F P)=1.6\end{array}$ & $\begin{array}{l}19(4.9 \%) w / A F P>400 \\
6(1.5 \%) w / \ln (A F P)=0 \\
\text { Median } \ln (A F P)=2.1\end{array}$ & $\begin{array}{l}22(2.9 \%) \text { w/AFP }>400 \\
11(1.5 \%) / \ln (\text { AFP })=0 \\
\text { Median } \ln (\text { AFP })=2.6\end{array}$ \\
\hline $\begin{array}{l}\text { Lab MELD } \\
+1.9^{*} \text { MELD to deMELD } \\
+1.143^{*} \text { MELD to MELD }{ }_{\mathrm{EQ}}\end{array}$ & $\begin{array}{l}\text { Range } 16-24 \\
0 \text { w/MELD = deMELD } \\
347(65.5 \%) w / M E L D=M E L D \\
E Q\end{array}$ & $\begin{array}{l}\text { Range } 14-18 \\
0 \text { w/MELD = deMELD } \\
345(88.5 \%) w / M E L D=M E L D \\
E Q\end{array}$ & $\begin{array}{l}\text { Range } 11-18 \\
585(78.3 \%) \text { w/MELD=deMELD } \\
0 \text { w/MELD=MELD } \\
\text { EQ }\end{array}$ \\
\hline
\end{tabular}

Table 8. Effect of covariates without score agreement. 


\section{Post-Transplant Survival}

Post-transplant survival was compared for alternative MELD scores in ranges 6-15, 16-21, 22-30, and 31-40; see Figure 10. A significant difference was seen between the lowest risk group and the 22-30 group under both scores $\left(p=0.007, M E L D_{E Q} ; p=0.015\right.$, deMELD; see Table 9). For the MELD $D_{E Q}$, this 22-

30 group has a steady decrease in survival rate for about two years and then nearly levels off, while for the deMELD this group has a slightly more gradual decrease over time. The highest risk group had a higher survival rate for the first year and then decreased quickly, while the highest risk group for deMELD had a sharper decrease in the first $1 \frac{1}{2}$ months and then decreased gradually over time. Data in this highest risk group were sparse under both scores as seen in the numbers at risk.

Cox proportional hazard models were also fit to predict post-transplant survival by these risk strata. The hazard ratio (HR) for the MELD ${ }_{E Q} 22-30$ group was $1.588(1.132,2.228, p=0.007)$, and $1.477(1.078,2.022, p=0.015)$ for deMELD 22-30 group, relative to the 6-15 groups. The HR's were not significant among the other strata as shown in Table 9, and proportional hazards assumptions were met (global chi-squared 5.669, $3 \mathrm{df}, \mathrm{p}=0.129$ for $M E L D_{\mathrm{EQ}}$; global chi-squared 3.7, $3 \mathrm{df}, \mathrm{p}=0.296$ for deMELD) ${ }^{28}$. 

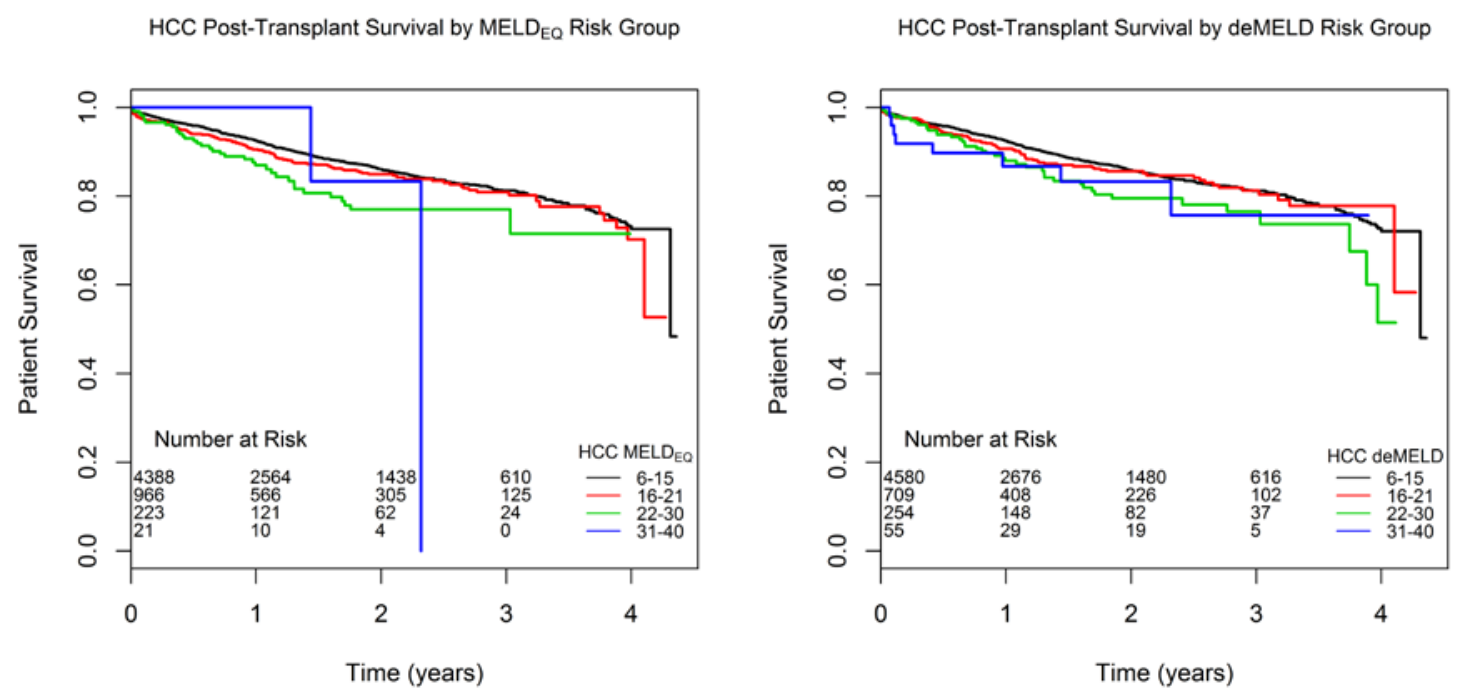

Figure 10. Post-transplant survival for HCC patients stratified by MELD $D_{E Q}$ and deMELD ranges. Kaplan-Meier survival curves for HCC patients with the indicated alternative MELD score ranges at last follow up prior to transplant. The number at risk in each stratum is shown for $0,1,2$, and 3 years at the bottom of each panel.

MELD $_{\mathrm{EQ}}$

\begin{tabular}{l|l|l|l}
\hline Group & HR & 95\% Cl & p-value \\
\hline$<16$ & reference & reference & reference \\
$16-21$ & 1.141 & $0.937,1.389$ & 0.189 \\
$22-30$ & 1.588 & $1.132,2.228$ & 0.007 \\
$31-40$ & 1.175 & $0.293,4.712$ & 0.820 \\
\hline
\end{tabular}

deMELD

\begin{tabular}{l|l|l|l}
\hline Group & HR & 95\% Cl & p-value \\
\hline$<16$ & reference & reference & reference \\
$16-21$ & 1.052 & $0.839,1.320$ & 0.661 \\
$22-30$ & 1.477 & $1.078,2.022$ & 0.015 \\
$31-40$ & 1.386 & $0.690,2.787$ & 0.359 \\
\hline
\end{tabular}

Table 9. Hazard Ratios for Post-Transplant Survival from Cox Proportional Hazard Models 


\section{DISCUSSION}

Previous research has demonstrated that scheduled progression of exception points is not an equitable method of prioritizing liver transplant between $\mathrm{HCC}$ and non-HCC patients. While the current allocation scheme implementing a six-month delay prior to granting exception points should improve equity of outcomes, reverting to scheduled exception point progression may still advantage HCC patients after six months and treats all HCC patients as having the same dropout risk regardless of tumor characteristics or laboratory MELD as discussed in Chapter 2. Hence it is unknown whether the current six-month delay provides the final solution for equitable transplant for $\mathrm{HCC}$ and non-HCC patients, and in the interim it is instructive to evaluate potential candidates for equivalent MELD scores to assess their strengths and weaknesses. The MELD $D_{E Q}$ and deMELD models both show improvement over scheduled progression of exception points, with better stratification of dropout and transplant probabilities. Overall numerical comparisons of the two scores slightly favored the MELD $\mathrm{EQ}_{\mathrm{Q}}$ for dropout prediction, with $\mathrm{C}$-indices of 0.678 and 0.664 respectively. However, this result was not statistically significant as $95 \%$ Cls for both $\mathrm{C}$-indices overlapped. Both scores match actual dropout probabilities comparably to laboratory MELD for non-HCC patients fairly well for patients with alternative MELD scores $<22$, though the deMELD remains more closely aligned for a longer time period than the MELD $\mathrm{EQ}_{\mathrm{EQ}}$ (approximately 8 months vs 6 months)

once a patient enters a given score range. Neither score matches actual dropout accurately for the higher risk groups with the exception of the MELD $D_{E Q}$ for those with scores 22-24. 
Differences in projected HCC dropout and non-HCC dropout were similar overall between the MELD $D_{E Q}$ and the deMELD. However, the projected dropout tended to be closer to non-HCC dropout under the deMELD for equivalent scores

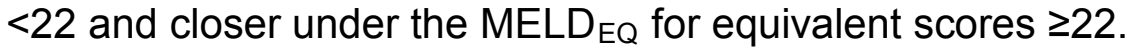

A problem for both scores is that dropout would increase beyond non$\mathrm{HCC}$ levels for groups with equivalent scores $<16$ if prioritization were based on them. However, the average difference over one year between projected dropout for these groups and actual dropout of analogous non-HCC groups is around 0.01 - near zero in the first few months since entering a given risk strata but increasing over time.

In contrast to the $<16$ groups, dropout is projected to decrease for higher risk groups, especially under the deMELD. This is because actual transplant and dropout probabilities for the higher risk groups are largely lower than those for corresponding non- $\mathrm{HCC}$ risk groups, so transplanting them at a higher rate to match non-HCC rates would be expected to decrease their dropout further.

In the original paper describing the MELD $\mathrm{EQ}^{15}$, actual dropout and transplant probabilities were depicted similarly to Figures $6 \mathrm{a}, 6 \mathrm{c}, 7 \mathrm{a}$ and $7 \mathrm{c}$ in this writing. These probabilities appear to have decreased in the newer data. A possible explanation may be that people are able to wait longer than they could in the past for transplant without dropping out, perhaps due to improvement in other therapies or patient management. Both scores can indirectly account for effects from other therapies (for example, ablation) if they are reassessed over time, since if a patient receives ablation and their maximum tumor size is reduced, their MELD $D_{E Q}$ and deMELD scores would also be reduced accordingly. 
This consideration was also discussed in the paper describing the deMELD ${ }^{13}$. Under the scheduled exception point progression, which resumes after six months with the recently implemented six-month delay, such a patient would receive the same number of exception points at each three-month interval as a patient who did not have another therapy (or any HCC patient who remained eligible for exception points). However, as ablation and other treatments are not covariates in either of the models evaluated, we did not directly look at their effects in this study.

Both scores improve risk stratification and allow for more equitable transplant probabilities. Although they do not match non-HCC transplant probabilities perfectly, they are much closer than under scheduled exception point progression for most risk groups. One notable difference between the two scores is that the deMELD identifies more patients in higher risk $(\geq 22)$ categories at listing. The main reasons for this are 1) the MELD $D_{\mathrm{EQ}}$ assigns 6.85 more points after six months on the list and 2) the deMELD gives much greater weight to tumor size (21.2 points for tumors $>1 \mathrm{~cm}$ vs. 1.194 points for each $\mathrm{cm}$ for the $\left.M E L D_{E Q}\right)$. The deMELD also uses discrete cutoff points for most of the HCC covariates while the MELD ${ }_{E Q}$ uses continuous values. Thus, the deMELD may be more "stable" compared to the MELD $D_{\mathrm{EQ}}$ for higher risk groups, in that a change in score requires a larger change in the covariates than it would for the MELD ${ }_{E Q}$.

Projected transplant under the deMELD matches non-HCC probabilities well for these higher risk groups. However, a potential concern with prioritizing higher risk HCC patients is earlier transplantation of patients with more 
aggressive tumors who may have poorer outcomes post-transplant. We did find that post-transplant survival is significantly lower for the 22-30 equivalent MELD score group compared to the lowest risk group for both scores, but no significant differences were found between the other risk groups. Adjustment of both scores may be warranted to avoid transplanting those at high risk for recurrence. This concern was mentioned in the original publication of the deMELD as well, and they suggested the possibility of using a 3-6 month waiting time along with their score ${ }^{13}$. Potential causes of lower post-transplant survival in the 22-30 group (e.g. recurrence vs. graft failure or other causes) were not investigated in this study. In addition, HCC dropout is already lower than that for non-HCC patients for groups with scores $\geq 22$, except for $M E L D_{E Q}>35$ after about 7 months from reaching this score. The lower dropout probabilities for scores in this range suggest that perhaps lower equivalent MELD scores could be more appropriate for some patients in this group. A possible area for future research could be simply modeling dropout of $\mathrm{HCC}$ and non-HCC patients based on laboratory MELD alone, and equating dropout hazards or probabilities to determine an appropriate adjustment to the MELD score for HCC patients.

One limitation of this study is that total risks/benefits between dropout, transplant, and post-transplant survival were not considered. Other investigators have discussed this ${ }^{16,19,20}$, and overall utility (combined dropout/post-transplant survival) is included in the model by Vitale et $\mathrm{al}^{14}$. Comparing that approach with those studied in this paper is another potential area for future research. However, the evaluation criteria for comparing the Vitale et al. score with the $M E L D_{E Q} / d e M E L D$ is difficult to determine given the differences in objectives 
between the studies. The covariates included in the models studied here have also been studied as prognostic indicators for post-transplant outcomes, particularly AFP ${ }^{4,19,29}$. Another limitation of the current study is that while setting time zero as the first time a patient enters a given risk category may be of greater clinical interest, it does not allow for projections for non-HCC patients under the proposed scoring schemes. However, comparison to actual non-HCC outcomes is useful for assessing how well the equivalent scores would improve equity between non-HCC and HCC transplant and dropout rates.

A strong point of this study is that although several models incorporating HCC characteristics for transplant prioritization have been proposed, no previous studies were identified that compared projected outcomes between two proposed HCC-specific models as well as with actual practice. While the current results do not provide a clear-cut answer to which of these scores better prioritizes HCC patients, they do highlight strengths and weaknesses of each which can provide direction for ongoing work on an HCC prioritization score.

In summary, the MELD $D_{E Q}$ and deMELD would both improve equity in transplant access between HCC and non-HCC patients compared to scheduled progression. While overall matching of projected dropout probabilities with nonHCC levels is nearly identical between the two scores, the deMELD does a better job of matching the lower risk scores $\leq 21$. An advantage of the $M E L D_{E Q}$ is that it "builds in" additional points at six months waitlist time and thus is a natural counterpart to the current six-month delay in granting exception points. For the deMELD, an advantage is that projected transplant probabilities are relatively close to those of non-HCC patients in most analogous MELD score ranges. 
Also, since the deMELD uses discrete cutoff points for most of the HCC covariates the score is unlikely to change dramatically over time. Disadvantages of both scores include projected dropout probabilities that exceed non-HCC probabilities for those with alternative MELD scores $<16$, and potential for prioritizing higher risk patients with poorer post-transplant survival and lower actual dropout rates. Thus further calibration and development of both scores is recommended before their implementation. 


\section{CHAPTER 4}

\section{SUMMARY}

Improvement in prioritization of liver transplant for patients with $\mathrm{HCC}$ is needed, as scheduled progression of exception points does not consider tumor characteristics and treats all HCC patients similarly regardless of their dropout risk. The possible solutions evaluated here do offer improvement, but each has limitations.

While the newly implemented six-month delay in assigning exception points to HCC patients will help address the disparity in transplant rates between $\mathrm{HCC}$ and non-HCC patients, the improvement will mostly be seen for the first six months as discussed in Chapter 2. After this time, transplant probabilities for HCC patients will quickly return to exceeding those for non-HCC patients and also become similar among $\mathrm{HCC}$ dropout risk strata.

The MELD $D_{E Q}$ would help equalize dropout probabilities between HCC and non-HCC patients over time, but would reduce transplant probabilities for HCC patients below those of non-HCC patients. Use of the MELD ${ }_{E Q}$ beginning at six months on the waiting list is projected to match non-HCC transplant probabilities well with very good risk stratification but may increase HCC dropout, especially for the highest risk group.

Comparison of the MELD $D_{E Q}$ and the deMELD scores, as shown in Chapter 3 , again revealed that both scores show promise but are projected to increase dropout probabilities beyond those of non-HCC patients in the same ranges for 
patients with equivalent scores $<16$. However, patients in this group currently have low dropout risk, and the projected increase above non-HCC dropout probabilities is not large for either score. For the higher risk groups (equivalent scores of at least 22), both scores would make transplant probabilities more equitable with non-HCC patients but then decrease dropout below that of nonHCC patients in the same risk groups, particularly for the deMELD. The deMELD assigns points heavily for tumor size, so a possible disadvantage is that patients scored higher by deMELD could be at risk for post-transplant recurrence. This study did find that patients in some high-risk groups may have worse posttransplant survival. This is also discussed in the original paper for the deMELD and the authors suggest possibly using a waiting period before use of the score $^{13}$. The MELD $E Q$ assigns more points for waitlist time of at least six months, but does not weight tumor size as heavily. Thus either score may have potential for use in conjunction with the six-month delay or after six months waitlist time. Alternatively, perhaps after further calibration, a score such as the deMELD or the MELD $D_{E Q}$ could replace the scheduled progression altogether. Further evaluation and research of these scores and other alternative prioritization schemes could aid in such calibration and refinement.

The methods of evaluating these prioritization schemes in the preceding chapters had several strengths and weaknesses. Somewhat different projection methods were used to evaluate the six-month delay and to compare the two equivalent MELD scores. Because relatively few patients are classified in the higher risk categories at listing, taking time zero as time a patient first enters a risk category, as described in Chapter 3, was more appropriate for comparing the 
two equivalent MELD scores. A limitation of using time first entering a risk state as time zero is that interpretation and comparison among risk groups may be less straightforward. Also, the projection method to include effects on non-HCC patients was not employed in the comparison study since it uses total number of organs transplanted at a given time and the proportions going to each stratum of $\mathrm{HCC}$ and non-HCC patients at a given time. Thus, the projections for the comparison in Chapter 3 only included those for HCC patients. One strength of this approach is that it can be more relevant clinically to determine a patient's probability of dropout from the time they develop a certain set of values of the relevant covariates.

For evaluating the six-month delay in Chapter 2, it was necessary to define time zero as the initial waitlist time, since the delay of six months is defined as time since listing. A limitation of using time since listing is that data can be sparse in the higher risk categories as mentioned earlier, since relatively few patients are classified in these higher risk groups at listing. An attempt to account for this was made by combining the higher risk categories for the sixmonth delay/MELD $\mathrm{EQ}_{\mathrm{EQ}}$ comparison. The projection method to include non-HCC patients has limitations as mentioned earlier in that it does not reflect calendar time for the whole group, so the estimates of available organs at each time may not be completely realistic. However, since prioritization schemes were compared using the same methods, it is still a useful comparison of the schemes. Overall, a strength of both studies described here is that projections were used to evaluate outcomes for $\mathrm{HCC}$ and non-HCC patients as whole groups as well as among different risk strata under three different prioritization 
methods. Additionally, this study identified strengths and weakness of each scheme that may help guide further research and improvement of the scores.

The MELD $D_{E Q}$ and the deMELD were selected for comparison for this project because they were derived most similarly, but evaluation of additional scoring methods, such as the method by Vitale et al that includes a utility score ${ }^{14}$ could be an area for future research. Evaluation of such scores using a model that considered both waitlist dropout and post-transplant mortality could be useful for determining net benefit. Additionally, further calibration of a score similar to the MELD $D_{E Q}$ or deMELD could be attempted by using simulated data to equate dropout and/or transplant outcomes between HCC and non-HCC patients. Further, exploration of new covariates that could influence waitlist dropout and post-transplant outcomes, such as other HCC-related biomarkers or response to other therapies for HCC, could be beneficial. Alternatively, equating dropout risk between $\mathrm{HCC}$ and non-HCC patients with MELD as the only covariate may help determine an appropriate and simple adjustment to the laboratory MELD for HCC patients.

In conclusion, transplant prioritization has not been equitable between $\mathrm{HCC}$ and non-HCC patients. Potential improvement has been made with implementing the six-month delay though it still may advantage HCC patients after 6 months. Possible solutions have been studied and proposed through alternative scoring schemes. While these improvements represent progress in decreasing this disparity, more progress is needed and further research on HCCspecific prioritization methods could help address this need. 


\section{REFERENCES}

1. Malinchoc M, Kamath PS, Gordon FD, Peine CJ, Rank J, ter Borg PC. A model to predict poor survival in patients undergoing transjugular intrahepatic portosystemic shunts. Hepatology. Apr 2000;31(4):864-871.

2. Kamath PS, Wiesner $\mathrm{RH}$, Malinchoc $\mathrm{M}$, et al. A model to predict survival in patients with end-stage liver disease. Hepatology. Feb 2001;33(2):464-470.

3. OPTN. Organ Procurement and Transplantation Network (OPTN) Policies, effective 10/8/2015.

4. Vincenzo Mazzaferro MD, Enrico Regalia, M.D., Roberto Doci, M.D., Salvatore Andreola , M.D., Andrea Pulvirenti , M.D., Federico Bozzetti , M.D., Fabrizio Montalto , M.D., Mario Ammatuna , M.D., Alberto Morabito , P H .D., and Leandro Gennari , M.D., Ph.D. Liver Transplantation for the Treatment of Small Hepatocellular Carcinomas in Patients With Cirrhosis. New England Journal of Medicine. 1996;334(11):693-699.

5. Washburn K, Edwards E, Harper A, Freeman R. Hepatocellular carcinoma patients are advantaged in the current liver transplant allocation system. American Journal of Transplantation. Jul 2010;10(7):1643-1648.

6. Freeman RB, Edwards EB, Harper AM. Waiting List Removal Rates Among Patients with Chronic and Malignant Liver Diseases. American Journal of Transplantation. 2006;6:1416-1421.

7. OPTN. Organ Procurement and Transplantation Network (OPTN) Policies, effective 2/19/2015.

8. Goldberg D, French B, Abt P, Feng S, Cameron AM. Increasing disparity in waitlist mortality rates with increased model for end-stage liver disease scores for candidates with hepatocellular carcinoma versus candidates without hepatocellular carcinoma. Liver Transpl. Apr 2012;18(4):434-443.

9. OPTN. OPTN/UNOS Policy Notice.December 12, 2014.

10. Heimbach JK, Hirose R, Stock PG, et al. Delayed hepatocellular carcinoma model for endstage liver disease exception score improves disparity in access to liver transplant in the United States. Hepatology. Jan 16 2015;61:1643-1650.

11. Piscaglia F, Camaggi V, Ravaioli M, et al. A new priority policy for patients with hepatocellular carcinoma awaiting liver transplantation within the model for end-stage liver disease system. Liver Transpl. Jun 2007;13(6):857-866.

12. Toso C, Dupuis-Lozeron E, Majno P, Berney $T$, Kneteman N, Perneger T, Morel P, Mentha G, Combescure C. A Model for Dropout Assessment of Candidates With or Without Hepatocellular Carcinoma On a Common Liver Transplant Waiting List. Hepatology. 2012;56:149-156.

13. Toso C, Majno P, Berney T, Morel P, Mentha G, Combescure C. Validation of a dropout assessment model of candidates with/without hepatocellular carcinoma on a common liver transplant waiting list. Transplant International. Jul 2014;27(7):686-695.

14. Vitale A, Volk ML, De Feo TM, et al. A method for establishing allocation equity among patients with and without hepatocellular carcinoma on a common liver transplant waiting list. Journal of Hepatology. Feb 2014;60(2):290-297. 
15. Marvin MR, Ferguson N, Cannon RM, Jones CM, Brock GN. MELD EQ : An alternative Model for End-Stage Liver Disease score for patients with hepatocellular carcinoma. Liver Transpl. May 2015;21(5):612-622.

16. Toso C, Mazzaferro V, Bruix J, Freeman R, Mentha G, Majno P. Toward a better liver graft allocation that accounts for candidates with and without hepatocellular carcinoma. American Journal of Transplantation. Oct 2014;14(10):2221-2227.

17. Bruix J, Sherman M, American Association for the Study of Liver D. Management of hepatocellular carcinoma: an update. Hepatology. Mar 2011;53(3):1020-1022.

18. Huo TI, Huang YH, Su CW, et al. Validation of the HCC-MELD for dropout probability in patients with small hepatocellular carcinoma undergoing locoregional therapy. Clinical transplantation. Jul-Aug 2008;22(4):469-475.

19. Pomfret EA, Washburn K, Wald C, et al. Report of a National Conference on Liver Allocation in Patients with Hepatocellular Carcinoma in the United States. Liver Transpl. Mar 2010;16(3):262-278.

20. Wedd JP, Nordstrom E, Nydam T, et al. Hepatocellular Carcinoma in Patients Listed for Liver Transplantation: Current and Future Allocation Policy and Management Strategies for the Individual Patient. Liver Transpl. Accepted manuscript online: 12 Oct 2015. doi: 10.1002/It.24356.

21. Ferguson N, Datta S, Brock G. msSurv: An R Package for Nonparametric Estimation of Multistate Models. Journal of Statistical Software. 2012;50(14).

22. Aalen O, Johansen S. An Empirical Transition Matrix for Non-homogeneous Markov Chains Based on Censored Observations Scandinavian Journal of Statistics. 1978;5(3):141-150.

23. Halazun K, Verna E, Samseith B, Guarrera J, Kato T, Brown R, Emond J. A Priority Pass to Death - Prioritization of Liver Transplant for HCC Worsens Survival. Am J Transplant. 2013;13(S5):S46.

24. Schlansky B, Chen Y, Scott DL, Austin D, Naugler WE. Waiting time predicts survival after liver transplantation for hepatocellular carcinoma: a cohort study using the United Network for Organ Sharing registry. Liver Transpl. Sep 2014;20(9):1045-1056.

25. Salvalaggio PR, Felga G, Axelrod DA, Della Guardia B, Almeida MD, Rezende MB. List and liver transplant survival according to waiting time in patients with hepatocellular carcinoma. Am J Transplant. Mar 2015;15(3):668-677.

26. Roayaie $\mathrm{K}$, Roayaie $\mathrm{S}$. Liver transplant for hepatocellular cancer: very small tumors, very large tumors, and waiting time. Clinics in Liver Disease. Aug 2014;18(3):603-612.

27. Harrell FE, Califf RM, Pryor DB, Lee KL, Rosati RA. Evaluating the Yield of Medical Tests. The Journal of the American Medical Association. 1982;247(18):2543-2546.

28. Grambsch PM, Therneau TM. Proportional Hazards Tests and Diagnostics Based on Weighted Residuals. Biometrika. 1994;81(3):515-526.

29. Clavien P-A, Lesurtel M, Bossuyt PMM, Gores GJ, Langer B, Perrier A. Recommendations for liver transplantation for hepatocellular carcinoma: an international consensus conference report. The Lancet Oncology. 2012;13(1):e11-e22. 


\section{CURRICULUM VITAE}

NAME: $\quad$ Sarah Katharine Alver

ADDRESS: $\quad 2305$ Hawthorne Ave

Louisville, KY 40205

DOB: $\quad$ Coupeville, WA - July 17, 1973

EDUCATION: $\quad$ Master of Science, Biostatistics

University of Louisville, 2013-2015

Bachelor of Science, Nutrition and Food Sciences

Utah State University, 2001

Cum Laude

\section{PROFESSIONAL EXPERIENCE:}

Clinical Dietitian, Compass Group, 2002-Present

Norton Healthcare, Louisville, KY: 2006 - Present

Jewish Hosp. Shelbyville, Shelbyville, KY: 2003-2006

Middle Tennessee Medical Center, Murfreesboro, TN: 2002-2003

Clinical Dietitian, Grays Harbor Comm. Hosp., Aberdeen, WA, 2001-2002

Dietetic Technician, United States Air Force Reserve, 1995-2001

Communications Security Analyst, United States Air Force, 1991-1995

CERTIFICATIONS: Registered Dietitian

Licensed Dietitian

Certified Nutrition Support Clinician

PROFESSIONAL MEMBERSHIPS:

Academy of Nutrition and Dietetics

American Society for Parenteral and Enteral Nutrition 